\title{
341.
}

\section{ON THE SEXTACTIC POINTS OF A PLANE CURVE.}

[From the Philosophical Transactions of the Royal Society of London, vol. CLv. (for the year 1865), pp. 545-578. Received November 5,-Read December 22, 1864.]

IT is, in my memoir "On the Conic of Five-pointic Contact at any point of a Plane Curve," Phil. Trans. vol. cxLIx. (1859), pp. 371-400, [261], remarked that as in a plane curve there are certain singular points, viz. the points of inflexion, where three consecutive points lie in a line, so there are singular points where six consecutive points of the curve lie in a conic; and such a singular point is there termed a "sextactic point." The memoir in question (here cited as "former memoir") contains the theory of the sextactic points of a cubic curve; but it is only recently that I have succeeded in establishing the theory for a curve of the order $m$. The result arrived at is that the number of sextactic points is $=m(12 m-27)$, the points in question being the intersections of the curve $m$ with a curve of the order $12 m-27$, the equation of which is

$$
\begin{aligned}
& \left(12 m^{2}-54 m+57\right) H \text { Jac. }\left(U, H, \Omega_{\bar{H}}\right) \\
& +(m-2)(12 m-27) H \text { Jac. }\left(U, H, \Omega_{\bar{U}}\right) \\
& +40(m-2)^{2} \quad \text { Jac. }(U, H, \Psi)=0 \text {, }
\end{aligned}
$$

where $U=0$ is the equation of the given curve of the order $m, H$ is the Hessian or determinant formed with the second differential coefficients $(a, b, c, f, g, h)$ of $U$, and, $(\mathfrak{A}, \mathfrak{B}, \mathfrak{C}, \mathfrak{F}, \mathfrak{G}, \mathfrak{S})$ being the inverse coefficients $\left(\mathfrak{A}=b c-f^{2}, \& c.\right)$, then

$$
\begin{aligned}
& \left.\Omega=(\mathfrak{A}, \mathfrak{B}, \mathfrak{E}, \mathfrak{F}, \mathfrak{S}), \mathfrak{S} \zeta \partial_{x}, \partial_{y}, \partial_{z}\right)^{2} H, \\
& \Psi=\left(\mathfrak{A}, \mathfrak{B}, \mathfrak{C}, \mathfrak{F}, \mathfrak{S}, \mathfrak{S} \nmid \partial_{x} H, \partial_{y} H, \partial_{z} H\right)^{2} \text {; }
\end{aligned}
$$


and Jac. denotes the Jacobian or functional determinant, viz.

$$
\text { Jac. }(U, H, \Psi)=\left|\begin{array}{lll}
\partial_{x} U, & \partial_{y} U, & \partial_{z} U \\
\partial_{x} H, & \partial_{y} H, & \partial_{z} H \\
\partial_{x} \Psi, & \partial_{y} \Psi, & \partial_{z} \Psi
\end{array}\right| \text {, }
$$

and Jac. ( $U, H, \Omega$ ) would of course denote the like derivative of $(U, H, \Omega)$; the subscripts $\left({ }_{H}, \bar{v}\right.$ ) of $\Omega$ denote restrictions in regard to the differentiation of this function, viz. treating $\Omega$ as a function of $U$ and $H$,

$$
\Omega=\left(\mathfrak{A}, \mathfrak{B}, \mathfrak{C}, \mathfrak{F}, \mathfrak{S}, \mathfrak{S} \chi a^{\prime}, b^{\prime}, c^{\prime}, f^{\prime}, 2 f^{\prime}, 2 g^{\prime}, 2 h^{\prime}\right)
$$

if $\left(a^{\prime}, b^{\prime}, c^{\prime}, f^{\prime}, g^{\prime}, h^{\prime}\right)$ are the second differential coefficients of $H$, then we have

$$
\begin{aligned}
& \partial_{x} \Omega=\left(\partial_{x} \mathfrak{H}, \ldots \gamma \quad a^{\prime}, \ldots\right) \quad\left(=\partial_{x} \Omega_{\bar{H}}\right) \\
& +\left(\stackrel{A}{\Re}, \ldots \gamma \partial_{x} a^{\prime}, \ldots\right) \quad\left(=\partial_{x} \Omega_{\bar{U}}\right) ;
\end{aligned}
$$

viz. in $\partial_{x} \Omega_{\bar{H}}$ we consider as exempt from differentiation $\left(a^{\prime}, b^{\prime}, c^{\prime}, f^{\prime}, g^{\prime}, h^{\prime}\right)$ which depend upon $H$, and in $\partial_{x} \Omega_{\bar{U}}$ we consider as exempt from differentiation $(\mathfrak{A}, \mathfrak{B}, \mathfrak{C}, \mathfrak{F}, \mathfrak{F}, \mathfrak{F})$ which depend upon $U$. We have similarly

$$
\partial_{y} \Omega=\partial_{y} \Omega_{\bar{H}}+\partial_{y} \Omega_{\bar{U}}, \text { and } \partial_{z} \Omega=\partial_{z} \Omega_{\bar{H}}+\partial_{z} \Omega_{\bar{U}}
$$

and in like manner

Jac. $(U, H, \Omega)=$ Jac. $\left(U, H, \Omega_{\bar{H}}\right)+$ Jac. $\left(U, H, \Omega_{\bar{U}}\right)$,

which explains the signification of the notations Jac. $\left(U, H, \Omega_{\bar{H}}\right)$, Jac. $\left(U, H, \Omega_{\bar{U}}\right)$.

The condition for a sextactic point is in the first instance obtained in a form involving the arbitrary coefficients $(\lambda, \mu, \nu)$; viz. we have an equation of the order 5 in $(\lambda, \mu, \nu)$ and of the order $12 m-22$ in the coordinates $(x, y, z)$. But writing $9=\lambda x+\mu y+\nu z$, by successive transformations we throw out the factors $9^{2}, 9,9,9$, thus arriving at a result independent of $(\lambda, \mu, \nu)$; viz. this is the before-mentioned equation of the order $12 m-27$. The difficulty of the investigation consists in obtaining the transformations by means of which the equation in its original form is thus divested of these irrelevant factors.

Articles Nos. 1 to 6.-Investigation of the Condition for a Sextactic Point.

1. Following the course of investigation in my former memoir, I take $(X, Y, Z)$ as current coordinates, and I write

$$
\Upsilon=(* \gamma X, Y, Z)^{m}=0
$$

for the equation of the given curve; $(x, y, z)$ are the coordinates of a particular point on the given curve, viz. the sextactic point; and $U,=(* \chi x, y, z)^{m}$, is what $\Upsilon$ becomes when $(x, y, z)$ are written in place of $(X, Y, Z)$ : we have thus $U=0$ as a condition satisfied by the coordinates of the point in question. 
2. Writing for shortness

$$
\begin{aligned}
& D U=\left(X \partial_{x}+Y \partial_{y}+Z \partial_{z}\right) U \\
& D^{2} U=\left(X \partial_{x}+Y \partial_{y}+Z \partial_{z}\right)^{2} U
\end{aligned}
$$

and taking $\Pi=a X+b Y+c Z=0$ for the equation. of an arbitrary line, the equation

$$
D^{2} U-\Pi D U=0
$$

is that of a conic having an ordinary (two-pointic) contact with the curve at the point $(x, y, z)$; and the coefficients of $\Pi$ are in the former memoir determined so that the contact may be a five-pointic one; the value obtained for $\Pi$ is

$$
\Pi=\frac{2}{3} \frac{1}{H} D H+\Lambda D U
$$

where

$$
\Lambda=\frac{1}{9 H^{3}}(-3 \Omega H+4 \Psi)
$$

3. This result was obtained by considering the coordinates of a point of the curve as functions of a single arbitrary parameter, and taking

$$
x+d x+\frac{1}{2} d^{2} x+\frac{1}{6} d^{3} x+\frac{1}{24} d^{4} x, y+\& c ., \quad z+\& c .
$$

for the coordinates of a point consecutive to $(x, y, z)$; for the present purpose we must go a step further, and write for the coordinates

$$
\begin{aligned}
& x+d x+\frac{1}{2} d^{2} x+\frac{1}{6} d^{3} x+\frac{1}{24} d^{4} x+\frac{1}{120} d^{5} x \\
& y+d y+\frac{1}{2} d^{2} y+\frac{1}{6} d^{3} y+\frac{1}{24} d^{4} y+\frac{1}{120} d^{5} y \\
& z+d z+\frac{1}{2} d^{2} z+\frac{1}{6} d^{3} z+\frac{1}{24} d^{4} z+\frac{1}{120} d^{5} z
\end{aligned}
$$

4. Hence if

$$
\partial_{1}=d x \partial_{x}+d y \partial_{y}+d z \partial_{z}, \quad \partial_{2}=d^{2} x \partial_{x}+d^{2} y \partial_{y}+d^{2} z \partial_{z}, \quad \& c .
$$

we have, in addition to the equations

$$
\begin{aligned}
& U=0, \\
& \partial_{1} U=0, \\
& \left(\partial_{1}{ }^{2}+2 \partial_{2}\right) U=0, \\
& \left(\partial_{1}{ }^{3}+3 \partial_{1} \partial_{2}+\partial_{3}\right) U=0, \\
& \left(\partial_{1}{ }^{4}+6 \partial_{1}{ }^{2} \partial_{2}+4 \partial_{1} \partial_{3}+3 \partial_{2}{ }^{2}+\partial_{4}\right) U=0,
\end{aligned}
$$

of my former memoir, the new equation

$$
\left(\partial_{1}{ }^{5}+10 \partial_{1}{ }^{3} \partial_{2}+10 \partial_{1}{ }^{2} \partial_{3}+15 \partial_{1} \partial_{2}{ }^{2}+5 \partial_{1} \partial_{4}+10 \partial_{2} \partial_{3}+\partial_{5}\right) U=0,
$$


and in addition to the equations, $(P=a x+b y+c z)$,

- $\quad(m-2) \partial_{1}{ }^{2} U+P \cdot \frac{1}{2} \partial_{1}{ }^{2} U=0$,

$-\frac{1}{3}\left[(m-1) \partial_{1}{ }^{3}+3(m-2) \partial_{1} \partial_{2}\right] U+P \cdot \frac{1}{6}\left(\partial_{1}{ }^{3}+3 \partial_{1} \partial_{2}\right) U+\partial_{1} P \cdot \frac{1}{2} \partial_{1}{ }^{2} U=0$,

$-\frac{1}{12}\left[(m-1)\left(\partial_{1}{ }^{4}+6 \partial_{1}{ }^{2} \partial_{2}\right)+(m-2)\left(4 \partial_{1} \partial_{3}+3 \partial_{2}{ }^{2}\right)\right] U$

$$
+P \cdot \frac{1}{24}\left(\partial_{1}{ }^{4}+6 \partial_{1}{ }^{2} \partial_{2}+4 \partial_{1} \partial_{3}+3 \partial_{2}{ }^{2}\right) U+\partial_{1} P \cdot \frac{1}{6}\left(\partial_{1}{ }^{3}+3 \partial_{1} \partial_{2}\right) U+\frac{1}{2} \partial_{2} P \cdot \frac{1}{2} \partial_{1}{ }^{2} U=0,
$$

giving in the first instance

$$
\begin{aligned}
P & =2(m-2), \\
\partial_{1} P & =\frac{2}{3} \partial_{1}{ }^{3} U \\
\partial_{1}{ }^{2} U & \\
\partial_{2} P & =\frac{1}{2} \frac{\left(\partial_{1}{ }^{4}+6 \partial_{1}{ }^{2} \partial_{2}\right) U}{\partial_{1}{ }^{2} U}-\frac{4}{9} \frac{\partial^{3} U}{\partial_{1}{ }^{2} U} \frac{\left(\partial_{1}{ }^{3}+3 \partial_{1} \partial_{2}\right) U}{\partial_{1}{ }^{2} U},
\end{aligned}
$$

and leading ultimately to the before-mentioned value of $\Pi$, we have the new equation

$$
\begin{aligned}
& -\frac{1}{60}\left[(m-1)\left(\partial_{1}{ }^{5}+10 \partial_{1}{ }^{3} \partial^{2}+10 \partial_{1}{ }^{2} \partial_{3}+15 \partial_{1} \partial_{2}{ }^{2}\right)+(m-2)\left(5 \partial_{1} \partial_{4}+10 \partial_{2} \partial_{3}\right)\right] U \\
& +P \cdot \frac{1}{120}\left(\partial_{1}{ }^{5}+10 \partial_{1}{ }^{3} \partial_{2}+10 \partial_{1}{ }^{2} \partial_{3}+15 \partial_{1} \partial_{2}{ }^{2} \quad+5 \partial_{1} \partial_{4}+10 \partial_{2} \partial_{3}\right) U \\
& +\partial_{1} P \cdot \frac{1}{24}\left(\partial_{1}{ }^{4}+6 \partial_{1}{ }^{2} \partial_{2}+4 \partial_{1} \partial_{3}+3 \partial_{2}{ }^{2}\right) U \\
& +\frac{1}{2} \partial_{2} P \cdot \frac{1}{6}\left(\partial_{1}{ }^{3}+3 \partial_{1} \partial_{2}\right) U \\
& +\frac{1}{6} \partial_{3} P \cdot \frac{1}{2} \partial_{1}{ }^{2} U=0 .
\end{aligned}
$$

5. This may be written in the form

$$
\begin{aligned}
& \text { - } \quad 2\left[(m-1)\left(\partial_{1}{ }^{5}+10 \partial_{1}{ }^{3} \partial_{2}+10 \partial_{1}{ }^{2} \partial_{3}+15 \partial_{1} \partial_{2}{ }^{2}\right)+(m-2)\left(5 \partial_{1} \partial_{4}+10 \partial_{2} \partial_{3}\right)\right] U \\
& +P\left(\quad \partial_{1}{ }^{5}+10 \partial_{1}{ }^{3} \partial_{2}+10 \partial_{1}{ }^{2} \partial_{3}+15 \partial_{1} \partial_{2}{ }^{2} \quad+5 \partial_{1} \partial_{4}+10 \partial_{2} \partial_{3}\right) U \\
& +5 \partial_{1} P\left(\quad \partial_{1}{ }^{4}+6 \partial_{1}{ }^{2} \partial_{2}+4 \partial_{1} \partial_{3}+3 \partial_{2}{ }^{2}\right) U \\
& +10 \partial_{2} P\left(\quad \partial_{1}{ }^{3}+3 \partial_{1} \partial_{2}\right) U \\
& +10 \partial_{3} P\left(\quad \partial_{1}{ }^{2} U\right)=0 \text {; }
\end{aligned}
$$

or putting for $P$ its value, $=2(m-2)$, the equation becomes

$$
\begin{aligned}
& -\quad 2\left(\partial_{1}{ }^{5}+10 \partial_{1}{ }^{3} \partial_{2}+10 \partial_{1}{ }^{2} \partial_{3}+15 \partial_{1} \partial_{2}{ }^{2}\right) U \\
& +5 \partial_{1} P\left(\partial_{1}{ }^{4}+6 \partial_{1}{ }^{2} \partial_{2}+4 \partial_{1} \partial_{3}+3 \partial_{2}{ }^{2}\right) U \\
& +10 \partial_{2} P\left(\partial_{1}{ }^{3}+3 \partial_{1} \partial_{2}\right) U \\
& +10 \partial_{3} P \cdot \partial_{2}{ }^{2} U=0
\end{aligned}
$$

or as this may also be written,

$$
\begin{aligned}
2\left(\partial_{1}{ }^{5}\right. & \left.+10 \partial_{1}{ }^{3} \partial_{2}+10 \partial_{1}{ }^{2} \partial_{3}+15 \partial_{1} \partial_{2}{ }^{2}\right) U \\
& +5 \partial_{1} P \cdot \partial_{4} U+10 \partial_{2} P \cdot \partial_{3} U+10 \partial_{3} P \cdot \partial_{2} U=0 .
\end{aligned}
$$


6. But the equation

$$
\Pi=\frac{2}{3} \frac{1}{H} D H+\Lambda D U,
$$

which is an identity in regard to $(X, Y, Z)$, gives

$$
\begin{aligned}
& \partial_{1} P=\frac{2}{3} \frac{1}{H} \partial_{1} H, \\
& \partial_{2} P=\frac{2}{3} \frac{1}{H} \partial_{2} H+\Lambda \partial_{2} U, \\
& \partial_{3} P=\frac{2}{3} \frac{1}{H} \partial_{3} H+\Lambda \partial_{3} U ;
\end{aligned}
$$

and substituting these values, the, foregoing equation becomes

$$
\begin{aligned}
2\left(\partial_{1}{ }^{5}\right. & \left.+10 \partial_{1}{ }^{3} \partial_{2}+10 \partial_{1}{ }^{2} \partial_{3}+15 \partial_{1} \partial_{2}{ }^{2}\right) U \\
& +\left(5 \partial_{4} U \partial_{1} H+10 \partial_{3} U \partial_{2} H+10 \partial_{2} U \partial_{3} H\right) \frac{2}{3} \frac{1}{H}+\Lambda .20 \partial_{2} U \partial_{3} U=0 ;
\end{aligned}
$$

or putting for $\Lambda$ its value, $=\frac{1}{9 H^{3}}(-3 \Omega H+4 \Psi)$, and multiplying by $\frac{9}{2} H^{2}$ this is

$$
\begin{aligned}
& 9 H^{2}\left(\partial_{1}{ }^{5}+10 \partial_{1}{ }^{3} \partial_{2}+10 \partial_{1}{ }^{2} \partial_{3}+15 \partial_{1} \partial_{2}{ }^{2}\right) U \\
&+ 15 H\left(\partial_{4} U \partial_{1} H+2 \partial_{3} U \partial_{2} H+2 \partial_{2} U \partial_{3} H\right) \\
&+\quad \frac{1}{H}(-3 \Omega H+4 \Psi) \cdot 10 \partial_{2} U \partial_{3} U=0,
\end{aligned}
$$

which is, in its original or unreduced form, the condition for a sextactic point.

Article Nos. 7 and 8.-Notations and Remarks.

7. Writing, as in my former memoir, $A, B, C$ for the first differential coefficients of $U$, we have $B \nu-C \mu, C \lambda-A \nu, A \mu-B \lambda$ for the values of $d x, d y, d z$, and instead of the symbol $\mathbf{D}$ used in my former memoir, I use indifferently the original symbol $\partial_{1}$, or write instead thereof $\partial$, to denote the resulting value

$$
\partial_{1}(=\partial)=(B \nu-C \mu) \partial_{x}+(C \lambda-A \nu) \partial_{y}+(A \mu-B \lambda) \partial_{z},
$$

and I remark here that for any function whatever $\Omega$, we have

$$
\partial \Omega=\left|\begin{array}{ccc}
A, & B, & C \\
\lambda, & \mu, & \nu \\
\partial_{x} \Omega, & \partial_{y} \Omega, & \partial_{z} \Omega
\end{array}\right|=\operatorname{Jac} .(U, \Omega, \Omega),
$$

where $9=\lambda x+\mu y+\nu z$. I write, as in the former memoir,

$$
\Phi=(\mathfrak{A}, \mathfrak{B}, \mathfrak{(}, \mathfrak{F}, \mathfrak{S}, \mathfrak{S})(\lambda, \mu, \nu)^{2} \text {; }
$$

C. $\mathbf{v}$. 
and also

$$
\nabla=\left(\mathfrak{A}, \mathfrak{B}, \mathfrak{(}, \mathfrak{F}, \mathfrak{S}, \mathfrak{S} \nmid \lambda, \mu, \nu \nmid \partial_{x}, \partial_{y}, \partial_{z}\right),
$$

which new symbol $\nabla$ serves to express the functions $\Pi, \square$, occurring in the former memoir; viz. we have $\Pi=2 \nabla \Phi, \square=2 \nabla H$, so that the symbols $\Pi$, $\square$ are not any longer required.

8. I remark that the symbols $\partial, \nabla$ are each of them a linear function of $\left(\partial_{x}, \partial_{y}, \partial_{z}\right)$, with coefficients which are functions of the variables $(x, y, z)$, and this being so, that for any function $\Pi$ whatever, we have

$$
\partial(\nabla \Pi)=(\partial . \nabla) \Pi+\partial \nabla \Pi,
$$

viz. in $\partial(\nabla \Pi)$ we operate with $\nabla$ on $\Pi$, thereby obtaining $\nabla \Pi$, and then with $\partial$ on $\nabla \Pi$; in $(\partial . \nabla) \Pi$ we operate with $\partial$ upon $\nabla$ in so far as $\nabla$ is a function of $(x, y, z)$, thus obtaining a new operating symbol $\partial . \nabla$, a linear function of $\left(\partial_{x}, \partial_{y}, \partial_{z}\right)$, and then operate with $\partial . \nabla$ upon $\Pi$; and lastly, in $\partial \nabla \Pi$, we simply multiply together $\partial$ and $\nabla$, thus obtaining a new operating symbol $\partial \nabla$ of the form $\left(\partial_{x}, \partial_{y}, \partial_{z}\right)^{2}$, and then operate therewith on $\Pi$; it is clear that, as regards the last-mentioned mode of combination, the symbols $\partial$ and $\nabla$ are convertible, or $\partial \nabla=\nabla \partial$, that is, $\partial \nabla \Pi=\nabla \partial \Pi$.

It is to be observed throughout the memoir that the point (.) is used (as above in $\hat{o} . \nabla$ ) when an operation is performed upon a symbol of operation as operand; the mere apposition of two or more symbols of operation (as above in $\partial \nabla$ ) denotes that the symbols of operation are simply multiplied together; and when $\partial \nabla$ is followed by a letter $\Pi$ denoting not a symbol of operation, but a mere function of the coordinates, that is in an expression such as $\partial \nabla \Pi$, the resulting operation $\partial \nabla$ is performed upon $\Pi$ as operand; if instead of the single letter $\Pi$ we have a compound symbol such as $H U$ or $H \nabla 9$, so that the expression is $\partial H U, \partial H \nabla 9, \partial \nabla H U$ or $\partial \nabla H \nabla 9$, then it is to be understood that it is merely the immediately following function $H$ which is operated upon by $\partial$ or $\partial \nabla$; in the few instances where any ambiguity might arise a special explanation is given.

\section{Article Nos. 9 to 11.-First transformation.}

9. We have, assuming always $U=0$, the following formulæ (see post, Article Nos. 31 to 33$)$ :

$$
\begin{aligned}
\left(\partial_{1}{ }^{5}+\right. & \left.10_{1}^{3} \partial_{2}+10 \partial_{1}{ }^{2} \partial_{3}+15 \partial_{1} \partial_{2}{ }^{2}\right) U \\
= & \frac{9^{2}}{(m-1)^{4}}\left\{\left(27 m^{2}-96 m+81\right) H \partial \Phi+\left(17 m^{2}-56 m+51\right) \Phi \partial H\right\} \\
& +\frac{9^{3}}{(m-1)^{4}}\{(-14 m-22)(\partial . \nabla) H-(10 m-18) \partial \nabla H\} \\
& +\frac{9^{4}}{(m-1)^{4}}\{\partial \Omega\},
\end{aligned}
$$




$$
\begin{aligned}
\partial_{4} U \partial_{1} H & +2 \partial_{3} U \partial_{2} H+2 \partial_{2} U \partial_{3} H \\
= & \frac{\lambda^{2}}{(m-1)^{4}}\left\{\left(-6 m^{2}+18 m-12\right) H^{2} \partial \Phi+\left(-17 m^{2}+60 m-55\right) H \Phi \partial \Phi\right\} \\
& +\frac{\lambda^{3}}{(m-1)^{4}}\{(2 m-2) H(\partial . \nabla) H+(8 m-16) \partial H \nabla H\} \\
& +\frac{\vartheta^{4}}{(m-1)^{4}}\{-\Omega \partial H\},
\end{aligned}
$$

$\partial_{2} U \partial_{3} U=\frac{9^{4}}{(m-1)^{4}} H \partial H$.

10. And by means of these the condition becomes

$$
\begin{aligned}
0= & \frac{9^{2} H^{2}}{(m-1)^{4}}\left\{\left(153 m^{2}-594 m+549\right) H \partial \Phi+\left(-102 m^{2}+396 m+366\right) \Phi \partial H\right\} \\
& +\frac{9^{3} H}{(m-1)^{4}}\{(-96 m+168) H(\partial . \nabla) H+(-90 m+162) H \partial \nabla H+(120 m-240) \partial H \nabla H\} \\
& +\frac{\Omega^{4}}{(m-1)^{4}}\left\{9 H^{2} \partial \Omega-45 H \Omega \partial H+40 \Psi \partial H\right\},
\end{aligned}
$$

being, as already remarked, of the degree 5 in the arbitrary coefficients $(\lambda, \mu, \nu)$, and of the order $12 m-22$ in the coordinates $(x, y, z)$.

11. But throwing out the factor $9^{2}$, and observing that in the first line the quadric functions of $m$ are each a numerical multiple of $51 m^{2}-198 m+183$, the condition becomes

$$
\begin{aligned}
0= & \left(51 m^{2}-198 m+183\right) H^{2}(3 H \partial \Phi-2 \Phi \partial H) \\
+ & 9\left\{(-96 m+168) H^{2}(\partial . \nabla) H+(-90 m+162) H^{2} \partial \nabla H+(120 m-240) \partial H \nabla H\right\} \\
+ & \Phi^{2}\left\{9 H^{2} \partial \Omega-45 H \Omega \partial H+40 \Psi \partial H\right\} .
\end{aligned}
$$

Article Nos. 12 and 13.-Second transformation.

12. We effect this by means of the formula

$$
(m-2)(3 H \partial \Phi-2 \Phi \partial H)=-9 \text { Jac. }(U, \Phi, H),
$$

for substituting this value of $(3 H \partial \Phi-2 \Phi \partial H)$ the equation becomes divisible by 9 and dividing out accordingly, the condition becomes

$$
\begin{aligned}
& -\frac{51 m^{2}-198 m+183}{m-2} H^{2} \text { Jac. }(U, \Phi, H) \\
& +(-96 m+168) H^{2}(\partial . \nabla) H+(-90 m+162) H^{2} \partial \nabla H+(120 m-240) H \partial H \nabla H \\
& +9\left(9 H^{2} \partial \Omega-45 H \Omega \partial H+40 \Psi \partial H\right)=0 .
\end{aligned}
$$

(J) here and elsewhere refers to the Jacobian Formula, see post, Article Nos. 34 and 35. 
13. We have (see post, Article Nos. 36 to 40 )

Jac. $(U, \Phi, H)=-(\partial . \nabla) H$;

and introducing also $\partial . \nabla H$ in place of $\partial \nabla H$ by means of the formula

$$
\partial \nabla H=\partial(\nabla H)-(\partial . \nabla) H
$$

the condition becomes

$$
\begin{aligned}
& \left\{\frac{51 m^{2}-198 m+183}{m-2}-(6 m-6)\right\} H^{2}(\partial . \nabla) H \\
+ & (-90 m+162) H^{2} \partial(\nabla H)+120(m-2) H \partial H \nabla H \\
+ & \neg\left(9 H^{2} \partial \Omega-45 H \Omega \partial H+40 \Psi \partial H\right)=0,
\end{aligned}
$$

or, as this may be written,

$$
\begin{aligned}
& \left(45 m^{2}-180 m+171\right) H^{2}(\partial . \nabla) H \\
+ & (-90 m+162)(m-2) H^{2} \partial(\nabla H)+120(m-2)^{2} H \partial H \nabla H \\
+ & (m-2) 9\left(9 H^{2} \partial \Omega-45 H \Omega \partial H+40 \Psi \partial H\right)=0 .
\end{aligned}
$$

Article Nos. 14 to 17.-Third transformation.

14. We have the following formulæ,

$$
\begin{aligned}
& \text { Jac. }(U, \nabla H, H)-(5 m-11) \partial H \nabla H+(3 m-6) H \partial(\nabla H)=0 \text {, } \\
& \text { ฯ Jac. }(U, \nabla, H) H-(2 m-4) \partial H \nabla H+(3 m-6) H(\partial . \nabla) H=0,
\end{aligned}
$$

in the latter of which, treating $\nabla$ as a function of the coordinates, we first form the symbol Jac. $(U, \nabla, H)$, and then operating therewith on $H$, we have Jac. $(U, \nabla, H) H$; these give

$$
\begin{gathered}
H \partial(\nabla H)=\frac{5 m-11}{3(m-2)} \partial H \nabla H-\frac{9}{3(m-2)} \text { Jac. }(U, \nabla H, H), \\
H(\partial . \nabla) H=\quad \frac{2}{3} \partial H \nabla H-\frac{9}{3(m-2)} \text { Jac. }(U, \nabla, H) H ;
\end{gathered}
$$

and substituting these values, the resulting coefficient of $H \partial H \nabla H$ is

$$
\begin{aligned}
&\left(45 m^{2}-180 m+171\right) \frac{2}{3} \\
&+(-90 m+162) \frac{5 m-11}{3} \\
&+120(m-2)^{2}
\end{aligned}
$$

which is $=0$. 
15. Hence the condition will contain the factor 9 , and throwing out this, and also the constant factor $\frac{1}{m-2}$, it becomes

$$
\begin{aligned}
& \left(-15 m^{2}+60 m-57\right) H \text { Jac. }(U, \nabla, H) H \\
+ & (30 m-54)(m-2) \quad H \text { Jac. }(U, \nabla H, H) \\
+ & (m-2)^{2}\left(9 H^{2} \partial \Omega-45 H \Omega \partial H+40 \Psi \partial H\right)=0 .
\end{aligned}
$$

16. We have

$$
\partial_{x}(\nabla H)=\left(\partial_{x} . \nabla\right) H+\partial_{x} \nabla H,
$$

viz. in $\left(\partial_{x} . \nabla\right) H$, treating $\nabla$ as a function of $(x, y, z)$ we operate upon it with $\partial_{x}$ to obtain the new symbol $\partial_{x} . \nabla$, and with this we operate on $H$; in $\partial_{x} \nabla$ we simply multiply together the symbols $\partial_{x}$ and $\nabla$, giving a new symbol of the form $\left(d_{x}^{2}, \partial_{x} \partial_{y}, \partial_{x} \partial_{z}\right)$ which then operates on $H$. We have the like values of $\partial_{y}(\nabla H)$ and $\partial_{z}(\nabla H)$; and thence also

$$
\text { Jac. }(U, \nabla H, H)=\text { Jac. }(U, \nabla, H) H+\text { Jac. }(U, \bar{\nabla} H, H) \text {, }
$$

viz. in the determinant Jac. $(U, \nabla, H)$ the second line corresponding to $\nabla$ is $\partial_{x} . \nabla$, $\partial_{y} . \nabla, \partial_{z} . \nabla$ ( $\nabla$ being the operand); and the Jacobian thus obtained is a symbol which operates on $H$ giving Jac. $(U, \nabla, H) H$; and in the determinant Jac. $(U, \nabla H, H)$ the second line is $\partial_{x} \nabla H, \partial_{y} \nabla H, \partial_{z} \nabla H$ ( $\nabla$ being simply multiplied by $\partial_{x}, \partial_{y}, \partial_{z}$ respectively).

17. Substituting, the condition becomes

$$
\begin{aligned}
& \left(-15 m^{2}+60 m-57\right) H \text { Jac. }(U, \nabla, H) H \\
+ & (30 m-54)(m-2)\{H \text { Jac. }(U, \nabla, H) H+\text { Jac. }(U, \nabla H, H)\} \\
+ & (m-2)^{2} \quad\left\{9 H^{2} \partial \Omega-54 H \Omega \partial H+40 \Psi \partial H\right\}=0,
\end{aligned}
$$

or, what is the same thing,

$$
\begin{aligned}
& \left(15 m^{2}-54 m+51\right) H \text { Jac. }(U, \nabla, H) H \\
+ & (30 m-54)(m-2) H \text { Jac. }(U, \bar{\nabla} H, H) \\
+ & (m-2)^{2}\left\{9 H^{2} \partial \Omega-4 \check{5} H \Omega \partial H+40 \Psi \partial H\right\}=0 .
\end{aligned}
$$

Article Nos. 18 to 27.-Fourth transformation, and final form of the condition for a Sextactic Point.

18. I write

$$
\begin{array}{rlrl}
(5 m-12) \Omega \partial H-(3 m-6) H \partial \Omega & =9 \text { Jac. }(U, \Omega, H) \\
\Omega \partial H+ & H \partial \Omega & = & \partial(\Omega H),
\end{array}
$$

and, introducing for convenience the new symbol $W$,

$$
-5 \Omega \partial H+\quad H \partial \Omega=W,
$$


so that

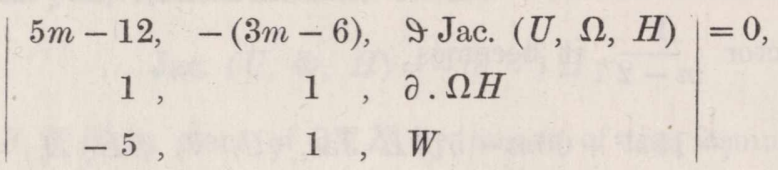

or, what is the same thing,

we have

$$
(8 m-18) W+6 \partial \text { Jac. }(U, \Omega, H)+(10 m-18) \partial(\Omega H)=0,
$$

$$
W=H \partial \Omega-5 \Omega \partial H=\frac{-3}{4 m-9} 9 \text { Jac. }(U, \Omega, H)-\frac{5 m-9}{4 m-9} \partial(\Omega H) .
$$

19. We have also

$$
(8 m-18) \Psi \partial H-(3 m-6) H \partial \Psi-9 \text { Jac. }(U, \Psi, H)=0 \text {, }
$$

that is

$$
\Psi \partial H=\quad \frac{\frac{1}{2}}{4 m-9} \text { 政. }(U, \Psi, H)+\frac{\frac{3}{2}(m-2)}{4 m-9} H \partial \Psi
$$

and thence

$$
\begin{aligned}
9 H W+40 \Psi \partial H= & 9 H^{2} \partial \Omega-45 H \Omega \partial H+40 \Psi \partial H \\
= & -\frac{9(5 m-9)}{4 m-9} H \partial(\Omega H)+\frac{60(m-2)}{4 m-9} H \partial \Psi \\
& +\frac{9}{4 m-9}\{-27 H \text { Jac. }(U, \Omega, H)+40 \text { Jac. }(U, \Psi, H)\} .
\end{aligned}
$$

20. The condition thus becomes

$$
\begin{aligned}
& \left(15 m^{2}-54 m+51\right)(4 m-9) H \text { Jac. }(U, \nabla, H) H \\
+ & 6(5 m-9)(m-2) \quad(4 m-9) H \text { Jac. }(U, \bar{\nabla} H, H) \\
+ & 3(m-2)\left\{-3(5 m-9)(m-2) H \partial(\Omega H)+20(m-2)^{2} H \partial \Psi\right\} \\
+ & (m-2)^{2} \curvearrowright\{-27 H \text { Jac. }(U, \Omega, H)+40 \text { Jac. }(U, \Psi, H)\}=0,
\end{aligned}
$$

which for shortness I represent by

$$
3 H \Pi+(m-2)^{2} 9\{-27 H \text { Jac. }(U, \Omega, H)+40 \text { Jac. }(U, \Psi, H)\}=0,
$$

so that we have

$$
\begin{aligned}
\Pi= & \left(5 m^{2}-18 m+17\right)(4 m-9) \operatorname{Jac} .(U, \nabla, H) H \\
& +2(5 m-9)(m-2)(4 m-9) \text { Jac. }(U, \bar{\nabla} H, H) \\
& +(m-2)\left\{-3(5 m-9)(m-2) \partial(\Omega H)+20(m-2)^{2} \partial \Psi\right\} .
\end{aligned}
$$

21. Write

$$
\left.\Psi_{1}=\left(\mathfrak{A}^{\prime}, \mathfrak{B}^{\prime}, \mathbb{E}^{\prime}, \mathscr{F}^{\prime}, \mathfrak{S H}^{\prime}, \mathfrak{S}^{\prime}\right\} A, B, C\right)^{2},
$$

where $(A, B, C)$ are as before the differential coefficients of $U$, and $\left(a^{\prime}, b^{\prime}, c^{\prime}, f^{\prime}, g^{\prime}, h^{\prime}\right)$ being the second differential coefficients of $H,\left(\mathfrak{U}^{\prime}, \mathfrak{B}^{\prime}, \mathfrak{l}^{\prime}, \mathfrak{F}^{\prime}, \mathfrak{F S}^{\prime}, \mathfrak{S}^{\prime}\right)$ are the inverse coefficients, viz., $\mathfrak{A}^{\prime}=b^{\prime} c^{\prime}-f^{\prime 2}$, \&c. We have

$-(m-1)^{2} \partial \Psi_{1}=(3 m-6)(3 m-7) \partial(\Omega H)-(3 m-7)^{2} \partial \Psi$ (see post, Nos. 41 to 46$)$, 
that is

$$
(3 m-6) \partial(\Omega H)=(3 m-7) \partial \Psi-\frac{(m-1)^{2}}{3 m-7} \partial \Psi_{1}
$$

and thence

$$
\begin{aligned}
\Pi= & \left(5 m^{2}-18 m+17\right)(4 m-9) \text { Jac. }(U, \nabla, H) H \\
& +2(5 m-9)(m-2)(4 m-9) \text { Jac. }(U, \bar{\nabla} H, H) \\
& +(m-2)\left\{\left(5 m^{2}-18 m+17\right) \partial \Psi+\frac{(m-1)^{2}(5 m-9)}{3 m-7} \partial \Psi_{1}\right\}=0 .
\end{aligned}
$$

22. Now

$\Psi=\left(\mathfrak{A}, \mathfrak{B}, \mathfrak{f}, \mathfrak{F}, \mathfrak{S F}, \mathfrak{S} \gamma A^{\prime}, B^{\prime}, C^{\prime}\right)^{2}, \quad \Psi_{1}=\left(\mathfrak{H}^{\prime}, \mathfrak{B}^{\prime},\left(\mathfrak{C}^{\prime}, \mathfrak{F}^{\prime}, \mathfrak{S H}^{\prime}, \mathfrak{S C}^{\prime}\right\} A, B, C\right)^{2}$, and writing for shortness

$$
\begin{aligned}
& E \Psi=\left(\partial \mathfrak{A}, \ldots \gamma A^{\prime}, B^{\prime}, C^{\prime}\right)^{2}, \quad F \Psi=\left(\mathfrak{U}, \ldots \chi A^{\prime}, B^{\prime}, C^{\prime} \chi \partial \mathfrak{A}^{\prime}, \partial \mathfrak{B}^{\prime}, \partial \mathfrak{\complement}^{\prime}\right) \text {, } \\
& E \Psi_{1}=\left(\partial \mathfrak{U}^{\prime}, \ldots \gamma A, B, C\right)^{2}, \quad F \Psi_{1}=\left(\mathfrak{I}^{\prime} \ldots \chi A, B, C \gamma \partial \mathfrak{U}, \partial \mathfrak{B}, \partial \mathfrak{\complement}\right) \text {, }
\end{aligned}
$$

(we might, in a notation above explained, write $E \Psi=\partial \Psi_{\bar{H}}, \quad F \Psi=\frac{1}{2} \partial \Psi_{\bar{U}}$, and in like manner $E \Psi_{1}=\partial \Psi_{1 \bar{U}}, F \Psi_{1}=\frac{1}{2} \partial \Psi_{1 \bar{H}}$ ), then we have

$$
\partial \Psi=E \Psi+2 F \Psi, \quad \partial \Psi_{1}=E \Psi_{1}+2 F \Psi_{1} .
$$

We have moreover

$$
\begin{aligned}
& \text { Jac. } \left.(U, \bar{\nabla} H, H)=-\frac{m-1}{3 m-7} E \Psi_{1},\right\} \text { post, Nos. } 47 \text { to } 50 \text {. } \\
& \text { Jac. }(U, \nabla, H) H=\quad-E \Psi,) \text { post, Nos. } 51 \text { to } \check{5} \text {. }
\end{aligned}
$$

23! The just-mentioned formulæ give

$$
\begin{aligned}
\Pi= & -\left(5 m^{2}-18 m+17\right)(4 m-9) E \Psi \\
& -2(5 m-9)(m-2)(4 m-9) \frac{m-1}{3 m-7} F \Psi_{1} \\
& +(m-2)\left(5 m^{2}-18 m+17\right)(E \Psi+2 F \Psi) \\
& +\frac{(5 m-9)(m-1)^{2}(m-2)}{3 m-7}\left(E \Psi_{1}+2 F \Psi_{1}\right)
\end{aligned}
$$

that is

$$
\begin{array}{rlr}
\Pi= & -(3 m-7)\left(5 m^{2}-18 m+17\right) & E \Psi \\
& +2(m-2)\left(5 m^{2}-18 m+17\right) & F \Psi \\
& +\frac{(5 m-9)(m-1)^{2}(m-2)}{3 m-7} & E \Psi_{1} \\
& -\frac{2(m-1)(m-2)(3 m-8)(5 m-9)}{3 m-7} F \Psi_{1},
\end{array}
$$


or, as this may also be written,

$$
\left.\begin{array}{rrr}
(3 m-7) \Pi=-\left(5 m^{2}-18 m+17\right) & \left\{-2(m-1)(m-2) F \Psi_{1}\right. & \left.+(3 m-7)^{2} E \psi\right\} \\
-(5 m-9)(m-2) & \{ & \left.(m-1)(3 m-8) F \Psi_{1}+(3 m-7)(3 m-8) F \Psi-(m-1)^{2} E \Psi_{1}\right\} \\
+\left(25 m^{2}-103 m+106\right)(m-2)\{ & -(m-1) F \Psi_{1} & (3 m-7) F^{h} \Psi
\end{array}\right\}
$$

24. But recollecting that

$$
\begin{aligned}
\Omega & =\left(\mathfrak{A}, \mathfrak{B}, \mathfrak{E}, \mathfrak{F}, \mathfrak{S}, \mathfrak{S} \gamma \partial_{x}, \partial_{y}, \partial_{z}\right)^{2} H \\
& =\left(\mathfrak{A}, \mathfrak{B}, \mathfrak{c}, \mathfrak{F}, \mathfrak{S}, \mathfrak{S} \gamma a^{\prime}, b^{\prime}, c^{\prime}, 2 f^{\prime}, 2 g^{\prime}, 2 h^{\prime}\right),
\end{aligned}
$$

and putting

$$
\begin{array}{ll}
E \Omega=\left(\partial \mathfrak{V}, \ldots \gamma \quad a^{\prime}, \ldots\right) & \left(=\partial \Omega_{\bar{H}}\right), \\
F \Omega=\left(\mathfrak{A}, \ldots \gamma \partial a^{\prime}, \ldots\right) & \left(=\partial \Omega_{\bar{U}}\right),
\end{array}
$$

we have, post, Nos. 41 to 46 ,

$$
\begin{array}{rrrr}
-2(m-1)(m-2) F \Psi_{1} & +(3 m-7)^{2} E \Psi_{1} & =(3 m-6)(3 m-7) H E \Omega, \\
(m-1)(3 m-8) F \Psi_{1}+(3 m-7)(3 m-8) F \Psi-(m-1)^{2} E \Psi_{2} & =(3 m-6)(3 m-7) H F \Omega, \\
-(m-1) F \Psi_{1} & (3 m-7) F \Psi- & (3 m-7) \Omega \partial H,
\end{array}
$$

and the foregoing equation becomes

$$
\begin{array}{rlr}
(3 m-7) \Pi= & -\left(5 m^{2}-18 m+17\right)(3 m-6) & (3 m-7) H E \Omega \\
& -(5 m-9)(m-2)(3 m-6) & (3 m-7) H F \Omega \\
& +(m-\Sigma)\left(25 m^{2}-103 m-106\right)(3 m-7) \Omega \partial H .
\end{array}
$$

25. But we have

$$
\begin{aligned}
& \text { จ Jac. }\left(U, H, \Omega_{\bar{H}}\right)-(3 m-6) H E \Omega+(2 m-4) \Omega \partial H=0, \\
& \text { ๆ Jac. }\left(U, H, \Omega_{\bar{U}}\right)-(3 m-6) H F \Omega+(3 m-6) \Omega \partial H=0,
\end{aligned}
$$

that is

$$
\begin{aligned}
& 3(m-2) H E \Omega=2(m-2) \Omega \partial H+9 \text { Jac. }\left(U, H, \Omega_{\bar{H}}\right), \\
& 3(m-2) H F \Omega=(3 m-8) \Omega \partial H+9 \text { Jac. }\left(U, H, \Omega_{\bar{U}}\right),
\end{aligned}
$$

and we thus obtain

$$
\begin{aligned}
\Pi= & -\left(5 m^{2}-18 m+17\right)\left\{2(m-2) \Omega \partial H+9 \text { Jac. }\left(U, H, \Omega_{\bar{H}}\right)\right\} \\
& -(5 m-9)(m-2)\left\{(3 m-8) \Omega \partial H+9 \text { Jac. }\left(U, H, \Omega_{\bar{U}}\right)\right\} \\
& +\left(25 m^{2}-103 m+106\right)(m-2) \Omega \partial H,
\end{aligned}
$$

where the coefficient of $(m-2) \Omega \partial H$ is

$$
\begin{aligned}
& -\left(10 m^{2}-36 m+34\right) \\
& -(5 m-9)(3 m-8) \\
& +\left(25 m^{2}-103 m+106\right)
\end{aligned}
$$


which is $=0$. Hence

$$
\begin{aligned}
\Pi= & -\left(5 m^{2}-18 m+17\right) \text { จ Jac. }\left(U, H, \Omega_{\bar{H}}\right) \\
& -(5 m-9)(m-2) \text { จ Jac. }\left(U, H, \Omega_{\bar{U}}\right) .
\end{aligned}
$$

26. Substituting this in the equation

$$
3 H \Pi+(m-2)^{2}\{-27 H \text { Jac. }(U, \Omega, H)+40 \text { Jac. }(U, \Psi, H)\}=0,
$$

the result contains the factor 9 , and, throwing this out, the condition is

$$
\begin{gathered}
3 H\left\{-\left(5 m^{2}-18 m+17\right) \text { Jac. }\left(U, H, \Omega_{\bar{H}}\right)-(5 m-9)(m-2) \text { Jac. }\left(U, H, \Omega_{\bar{U}}\right)\right\} \\
+(m-2)^{2}\{27 H \text { Jac. }(U, H, \Omega)-40 \text { Jac. }(U, H, \Psi)\}=0,
\end{gathered}
$$

or, as this may also be written,

$$
\begin{array}{lrr}
-\left(15 m^{2}-54 m+51\right) H \text { Jac. }\left(U, H, \Omega_{\bar{H}}\right)-3(5 m-9)(m-2) H \text { Jac. }\left(U, H, \Omega_{\bar{U}}\right) \\
+27(m-2)^{2} & \left\{H \text { Jac. }\left(U, H, \Omega_{\bar{H}}\right)+\right. & \left.H \text { Jac. }\left(U, H, \Omega_{\bar{U}}\right)\right\} \\
-40(m-2)^{2} & \text { Jac. }(U, H, \Psi)=0 . &
\end{array}
$$

27. Hence the condition finally is

$$
\begin{array}{r}
\left(12 m^{2}-54 m+57\right) H \text { Jac. }\left(U, H, \Omega_{\bar{H}}\right)+(m-2)(12 m-27) H \text { Jac. }\left(U, H, \Omega_{\bar{U}}\right) \\
-40(m-2)^{2} \text { Jac. }(U, H, \Psi)=0,
\end{array}
$$

or, as this may also be written,

$$
\begin{array}{r}
-3(m-1) H \text { Jac. }\left(U, H, \Omega_{\bar{H}}\right)+(m-2)(12 m-27) H \text { Jac. }(U, H, \Omega) \\
-40(m-2)^{2} \text { Jac. }(U, H, \Psi)=0,
\end{array}
$$

viz. the sextactic points are the intersections of the curve $m$ with the curve represented by this equation; and observing that $U, H, H \Omega$ and $\Psi$ are of the orders $m, 3 m-6$, $8 m-18$ respectively, the order of the curve is as above mentioned $=12 m-27$.

\section{Article Nos. 28 to 30.-Application to a Cubic.}

28. I have in my former memoir, No. 30, shown that for a cubic curve

$$
\Omega\left(\mathfrak{A}, \mathfrak{B}, \mathfrak{E}, \mathfrak{F}, \mathfrak{S}, \mathfrak{S} \gamma \partial_{x}, \partial_{y}, \partial_{z}\right)^{2} H=-2 S . U=0 \text {; }
$$

this implies Jac. $(U, H, \Omega)=0$, and hence if one of the two Jacobians, Jac. (U, $\left.H, \Omega_{U}\right)$, Jac. $\left(U, H, \Omega_{\bar{H}}\right)$ vanish, the other will also vanish. Now, using the canonical form

we have

$$
U=x^{3}+y^{3}+z^{3}+6 l x y z
$$

$$
\begin{aligned}
\Omega= & \left(\mathfrak{A}, \ldots \gamma a^{\prime}, \ldots\right) \\
= & \left(y z-l^{2} x^{2}, \quad z x-l^{2} y^{2}, \quad x y-l^{2} z^{2}, \quad l^{2} y z-l x^{2}, \quad l^{2} z x-l y^{2}, \quad l^{2} x y-l z^{2}\right) \\
& \left(\quad-3 l^{2} x, \quad-3 l^{2} y, \quad-3 l^{2} z, \quad\left(1+2 l^{3}\right) x, \quad\left(1+2 l^{3}\right) y, \quad\left(1+2 l^{3}\right) z\right)
\end{aligned}
$$

C. V. 
the development of which in fact gives the last-mentioned result. But applying this formula to the calculation of Jac. $\left(U, H, \Omega_{\bar{U}}\right)$, then disregarding numerical factors, we have

$$
\begin{aligned}
\partial_{x} \Omega_{\bar{U}}= & \left(y z-l^{2} x^{2}, ., . l^{2} y z-l x^{2}, ., . \chi-3 l^{2}, 0,0,\left(1+2 l^{3}\right), 0,0\right) \\
= & -3 l^{2} \quad\left(y z-l^{2} x^{2}\right) \\
& +\left(1+2 l^{3}\right)\left(l^{2} y z-l x^{2}\right) \\
= & \left(-l+l^{4}\right)\left(x^{2}+2 l y z\right),=S \partial_{x} U
\end{aligned}
$$

and in like manner $\partial_{y} \Omega_{\bar{U}}=S \partial_{y} U, \quad \partial_{z} \Omega_{\bar{U}}=S \partial_{z} U$, and therefore

Jac. $\left(U, H, \Omega_{\bar{U}}\right)=S$ Jac. $(U, H, U)=0$,

whence also

$$
\text { Jac. }\left(U, H, \Omega_{\dot{H}}\right)=0 \text {; }
$$

and the condition for a sextactic point assumes the more simple form,

Jac. $(U, H, \Psi)=0$.

29. Now (former memoir, No. 32) we have

$$
\begin{aligned}
& \Psi=(\mathfrak{A}, \mathfrak{B}, \mathfrak{C}, \mathfrak{F}, \mathfrak{B}, \mathfrak{S})\left(\partial_{x} H, \partial_{y} H, \partial_{z} H\right)^{2} \\
& =\left(1+8 l^{3}\right)^{2} \quad\left(y^{3} z^{3}+z^{3} x^{3}+x^{3} y^{3}\right) \\
& +\left(-9 l^{6}\right) \quad\left(x^{3}+y^{3}+z^{3}\right)^{2} \\
& +\left(-2 l-5 l^{4}-20 l^{7}\right)\left(x^{3}+y^{3}+z^{3}\right) x y z \\
& +\left(-15 l^{2}-78 l^{5}+12 l^{8}\right) x^{2} y^{2} z^{2},
\end{aligned}
$$

or observing that $x^{3}+y^{3}+z^{3}$ and $x y z$, and therefore the last three lines of the expression of $\Psi$ are functions of $U\left(=x^{3}+y^{3}+z^{3}+6 l x y z\right)$ and $H\left(=-l^{2}\left(x^{3}+y^{3}+z^{3}\right)+\left(1+2 l^{3}\right) x y z\right)$, and consequently give rise to the term $=0$ in Jac. $(U, H, \Psi)$, we may write

$$
\Psi=\left(1+8 l^{3}\right)^{2}\left(y^{3} z^{3}+z^{3} x^{3}+x^{3} y^{3}\right) .
$$

30. We have then, disregarding a constant factor,

Jac. $(U, H, \Psi)=$ Jac. $\left(x^{3}+y^{3}+z^{3}, x y z, y^{3} z^{3}+z^{3} x^{3}+x^{3} y^{3}\right)$,

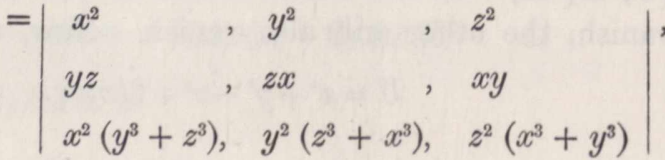

$$
\begin{aligned}
& =x^{3}\left(y^{6}-z^{6}\right)+y^{3}\left(z^{6}-x^{6}\right)+z^{3}\left(x^{6}-y^{6}\right), \\
& =\left(y^{3}-z^{3}\right)\left(z^{3}-x^{3}\right)\left(x^{3}-y^{3}\right),
\end{aligned}
$$


so that the sextactic points are the intersections of the curve

with the curve

$$
U=x^{3}+y^{3}+z^{3}+6 l x y z=0,
$$

$$
\left(y^{3}-z^{3}\right)\left(z^{3}-x^{3}\right)\left(x^{3}-y^{3}\right)=0 \text {. }
$$

Article Nos. 31 to 33.-Proof of identities for the first transformation.

31. Calculation of $\left(\partial_{1}{ }^{5}+10 \partial_{1}{ }^{3} \partial_{2}+10 \partial_{1}{ }^{2} \partial_{3}+15 \partial_{1} \partial_{2}{ }^{2}\right) U$.

Writing $\partial$ in place of $\mathbf{D}$, we have (former memoir, No. 20)

$$
\left(\partial_{1}^{4}+6 \partial_{1}{ }^{2} \partial_{2}\right) U=\frac{\partial^{2}}{(m-1)^{2}}\left(-2 \partial_{2} H-\partial^{2} H+\frac{3 m-6}{m-1} H \Phi-\frac{29}{m-1} \nabla H\right) .
$$

But

$$
\left.\begin{array}{l}
-2 \partial_{2} H=\frac{6 m-12}{m-1} H \Phi-\frac{29}{m-1} \nabla H \\
-\partial^{2} H=\frac{(3 m-6)(3 m-7)}{(m-1)^{2}} H \Phi-\frac{6 m-14}{(m-1)^{2}} 9 \nabla H+\frac{9^{2}}{(m-1)} \Omega,
\end{array}\right\} \begin{aligned}
& \text { former memoir } \\
& \text { Nos. } 21 \text { and } 22 ;
\end{aligned}
$$

and thence

$$
\begin{aligned}
\left(\partial_{1}{ }^{4}+6 \partial_{1}{ }^{2} \partial_{2}\right) U= & \frac{9^{2}}{(m-1)^{4}}\left(18 m^{2}-66 m+60\right) H \Phi \\
& +\frac{9^{3}}{(m-1)^{4}}(-10 m+18) \nabla H \\
& +\frac{9^{4}}{(m-1)^{4}}(\Omega)
\end{aligned}
$$

whence operating on each side with $\partial_{1},=\partial$, we have

$$
\begin{aligned}
\left(\partial_{1}{ }^{5}+10 \partial_{1}{ }^{3} \partial_{2}+6 \partial_{1}{ }^{2} \partial_{3}+12 \partial_{1} \partial_{2}{ }^{2}\right) U= & \frac{9^{2}}{(m-1)^{4}}\left(18 m^{2}-66 m+60\right)(H \partial \Phi+\Phi \partial H) \\
& +\frac{9^{3}}{(m-1)^{4}}(-10 m+18)\{(\partial . \nabla) H+\partial \nabla H\} \\
& +\frac{9^{4}}{(m-1)^{4}} \partial \Omega .
\end{aligned}
$$

We have besides (see Appendix, Nos. 69 to 74),

$$
\begin{aligned}
\partial_{1}{ }^{2} \partial_{3} U= & \frac{9^{2}}{(m-1)^{3}}\{(3 m-6) H \partial \Phi+(-m+3) \Phi \partial H\} \\
& +\frac{9^{3}}{(m-1)^{3}}\{-(\partial . \nabla) H\}, \\
\partial_{1} \partial_{2}{ }^{2} U= & \frac{9^{2}}{(m-1)^{2}}(-H \partial \Phi+\Phi \partial H) ;
\end{aligned}
$$


and thence

$$
\begin{aligned}
\left(4 \partial_{1}{ }^{2} \partial_{3}+3 \partial_{1} \partial_{2}{ }^{2}\right) U= & \frac{9^{2}}{(m-1)^{3}}\{(90 m-21) H \partial \Phi+(-m+9) \Phi \partial H\} \\
& +\frac{\vartheta^{3}}{(m-1)^{3}}\{-4(\partial . \nabla) H\} ;
\end{aligned}
$$

and adding this to the foregoing expression for $\left(\partial_{1}{ }^{5}+10 \partial_{1}{ }^{3} \partial_{2}+6 \partial_{1}{ }^{2} \partial_{3}+12 \partial_{1} \partial_{2}{ }^{2}\right) \dot{U}$, we have $\left(\partial_{1}^{5}+10 \partial_{1}^{3} \partial_{2}+10 \partial_{1}^{2} \partial_{3}+15 \partial_{1} \partial_{2}^{2}\right) U=$

$$
\begin{aligned}
& \frac{\Omega^{2}}{(m-1)^{4}}\left\{\left(27 m^{2}-96 m+81\right) H \partial \Phi+\left(17 m^{2}-56 m+51\right) \Phi \partial H\right\} \\
+ & \frac{9^{3}}{(m-1)^{4}}\{(-14 m+22)(\partial . \nabla) H+(-10 m+18) \partial \nabla . H\} \\
+ & \frac{9^{4}}{(m-1)^{4}} \partial \Omega .
\end{aligned}
$$

32. Calculation of

$$
\partial_{4} U \partial_{1} H+2 \partial_{3} U \partial_{2} H+2 \partial_{2} U \partial_{3} H
$$

We have

$$
\begin{array}{l|l}
\partial_{4} U=\frac{9^{2}}{(m-1)^{2}} \frac{2}{3} \partial_{2} H+\partial^{2} H-\frac{1}{m-1} H \Phi-\frac{\frac{2}{3} \vartheta}{m-1} \nabla H, & \partial_{1} H=\partial H \\
\partial_{3} U=\frac{\lambda^{2}}{(m-1)^{2}} \partial H, & \partial_{2} H=\partial_{2} H \\
\partial_{2} U=\frac{\vartheta^{2}}{(m-1)^{2}} H, & \partial_{3} H=\frac{1}{m-1}(-3 m+6) \partial \Phi-\Phi \partial H+\frac{9}{m-1}(\partial . \nabla) H,
\end{array}
$$

for which values see Appendix, No. 58. And hence the expression sought for is

$$
\begin{aligned}
=\frac{\mathfrak{Q}^{2}}{(m-1)^{3}}\{ & \left((m-1)\left(\frac{2}{3} \partial_{2} H+\partial^{2} H\right)-H \Phi-\frac{2}{3} \vartheta \nabla H\right) \partial H \\
& +2(m-1) \partial H \partial_{2} H \\
& +2 H((-3 m+6) H \partial \Phi-\Phi \partial H+\Phi(\partial . \nabla) H)\},
\end{aligned}
$$

which is

$$
\begin{aligned}
&=\frac{9^{2}}{(m-1)^{3}}\{ \frac{8}{3}(m-1) \partial H \partial_{2} H \\
&+(m-1) \partial H \partial^{2} H \\
&+\left.(-6 m+12) H^{2} \partial \Phi-3 H \Phi \partial H\right\} \\
&+\frac{9^{3}}{(m-1)^{3}}\left\{2 H(\partial . \nabla) H-\frac{2}{3} \partial H \nabla H\right\} .
\end{aligned}
$$


But we have, former memoir, Nos. 21 and 25,

$$
\begin{aligned}
& \partial_{2} H=-\frac{(3 m-6)}{m-1} H \Phi-\frac{9}{m-1} \nabla H, \\
& \partial^{2} H=-\frac{(3 m-6)(3 m-7)}{(m-1)^{2}} H \Phi+\frac{6 m-14}{(m-1)^{2}} \nabla \nabla H-\frac{9^{2}}{(m-1)^{2}} \Omega,
\end{aligned}
$$

so that the foregoing expression becomes

$$
\begin{aligned}
& =\frac{\unlhd^{2}}{(m-1)^{3}}\left\{-(8 m-16) H \Phi \partial H+\frac{8}{3} 9 \partial H \nabla H\right. \\
& -\frac{(3 m-6)(3 m-7)}{m-1} H \Phi \partial H+\frac{6 m-14}{m-1}-2 \partial H \nabla H-\frac{9^{2}}{m-1} \Omega \partial H \\
& \left.3 H \Phi \partial H-(6 m-12) H^{2} \partial \Phi\right\} \\
& +\frac{\partial^{3}}{(m-1)^{3}}\left\{2 H(\partial . \nabla) H-\frac{2}{3} \partial H \nabla H\right\}
\end{aligned}
$$

or finally

$$
\begin{aligned}
& \partial_{4} U \partial_{1} H+2 \partial_{3} U \partial_{2} H+2 \partial_{2} U \partial_{3} H= \\
& \quad \frac{\partial^{2}}{(m-1)^{4}}\left\{\left(-6 m^{2}+18 m-12\right) H^{2} \partial \Phi+\left(-17 m^{2}+60 m-\check{\partial}\right) H \Phi \partial H\right\} \\
& +\frac{\vartheta^{3}}{(m-1)^{4}}\{(2 m-2) H(\partial \cdot \nabla) H+(8 m-16) \partial H \nabla H\} \\
& +\frac{\vartheta^{4}}{(m-1)^{4}}\{-\Omega \partial H\} .
\end{aligned}
$$

33. Calculation of $\partial_{2} U \partial_{3} U$.

This is

$$
=\frac{9^{4}}{(m-1)^{4}} H \partial H
$$

Article Nos. 34 and 35.-The Jacobian Formula.

34. In general, if $P, Q, R, S$ be functions of the degrees $p, q, r, s$ respectively, we have identically

$$
\begin{array}{cccc}
p P, & q Q, & r R, & s S \\
\partial_{x} P, & \partial_{x} Q, & \partial_{x} R, & \partial_{x} S \\
\partial_{y} P, & \partial_{y} Q, & \partial_{y} R, & \partial_{y} S \\
\partial_{z} P, & \partial_{z} Q, & \partial_{z} R, & \partial_{z} S
\end{array} \mid=0
$$

or, what is the same thing,

$p P$ Jac. $(Q, R, S)-q Q$ Jac. $(R, S, P)+r R$ Jac. $(S, P, Q)-s S$ Jac. $(P, Q, R)=0$. 
Hence in particular if $P=\vec{U}$, and assuming $U=0$, we have

$-q Q$ Jac. $(R, S, U)+r R$ Jac. $(S, U, Q)-s S$ Jac. $(U, Q, R)=0$.

If moreover $Q=9$, and therefore $q=1$, we have

-9 Jac. $(R, S, U)+r R$ Jac. $(S, U, 9)-s S$ Jac. $(U, 9, R)=0$;

or, as this may also be written,

-9 Jac. $(U, R, S)+r R$ Jac. $(U, 9, S)-s S$ Jac. $(U, 9, R)=0$;

that is

-9 Jac. $(U, R, S)+r R \partial S-s S \partial R=0$.

35. Particular cases are

$$
\begin{aligned}
& (2 m-4) \Phi \partial H-(3 m-6) H \partial \Phi=9 \text { Jac. }(U, \Phi, H) \text {, ante, No. 12, } \\
& (5 m-11) \nabla H \partial H-(3 m-6) H \partial(\nabla H)=9 \text { Jac. }(U, \nabla H, H), \quad \text { " 14, } \\
& (2 m-4) \nabla: \partial H-(3 m-6) H \partial . \nabla=9 \text { Jac. }(U, \nabla, H), \quad, \quad \text { ” } \\
& (5 m-12) \Omega \partial H-(3 m-6) H \partial \Omega \quad=9 \mathrm{Jac} .(U, \Omega, H), \quad \text {, 18, } \\
& (8 m-18) \Psi \partial H-(3 m-6) H \partial \Psi \quad=9 \text { Jac. }(U, \Psi, H), \quad \text { " } 19 \text {, } \\
& (2 m-4) \quad \Omega \partial H-(3 m-6) H E \Omega \quad=9 \operatorname{Jac} .\left(U, \Omega_{\bar{H}}, H\right), \quad \text { " 25, } \\
& (3 m-8) \quad \Omega \partial H-(3 m-6) H F \Omega \quad=9 \text { Jac. }\left(U, \Omega_{\bar{U}}, H\right), \quad \text { " " }
\end{aligned}
$$

where it is to be observed that in the third of these formulæ $I$ have, in accordance with the notation before employed, written $\partial . \nabla$ to denote the result of the operation $\partial$ performed on $\nabla$ as operand. I have also written $\nabla: \partial H$ to show that the operation $\nabla$ is not to be performed on the following $\partial H$ as an operand, but that it remains as an unperformed operation. As regards the last two equations, it is to be remarked that the demonstration in the last preceding number depends merely on the homogeneity of the functions, and the orders of these functions: in the former of the two formulæ, the differentiation of $\Omega$ is performed upon $\Omega$ in regard to the coordinates $(x, y, z)$ in so far only as they enter through $U$, and $\Omega$ is therefore to be regarded as a function of the order $2 m-4$; in the latter of the two formulæ the differentiation is to be performed in regard to the coordinates in so far only as they enter through $H$, and $\Omega$ is therefore to be regarded as a function of the order $3 m-8$. The two formulæ might also be written

$$
\begin{aligned}
& (2 m-4) \Omega \partial H-(3 m-6) H \partial \Omega_{\bar{H}}=9 \text { Jac. }\left(U, \Omega_{\bar{H}}, H\right), \\
& (3 m-8) \Omega \partial H-(3 m-6) H \partial \Omega_{\bar{U}}=9 \text { Jac. }\left(U, \Omega_{\bar{U}}, H\right) ;
\end{aligned}
$$

and it may be noticed that, adding these together, we obtain the foregoing formula,

$$
(5 m-12) \Omega \partial H-(3 m-6) H \partial \Omega=9 \text { Jac. }(U, \Omega, H) .
$$


Article Nos. 36 to 40.-Proof of equation (ว. $\nabla) H=$ Jac. $(U, H, \Phi)$, used in the second transformation.

36. We have

$$
\begin{aligned}
& \nabla=\left(\mathfrak{A}, . . \chi \lambda, \mu, \nu \gamma \partial_{x}, \partial_{y}, \partial_{z}\right) \\
& =\left(\mathfrak{A} \partial_{x}+\mathfrak{S} \partial_{y}+\mathfrak{S} \partial_{z}, \mathfrak{W} \partial_{x}+\mathfrak{B} \partial_{y}+\mathfrak{F} \partial_{z}, \mathbb{S} \partial_{x}+\mathfrak{F} \partial_{y}+\left(\mathfrak{E} \partial_{z}\right) \lambda, \mu, \nu\right) \text {. }
\end{aligned}
$$

Also

$$
\begin{aligned}
\partial & =(B \nu-C \mu) \partial_{x}+(C \lambda-A \nu) \partial_{y}+(A \mu-B \lambda) \partial_{z} \\
& =\lambda P+\mu Q+\nu R,
\end{aligned}
$$

if for a moment $P, Q, R=C \partial_{y}-B \partial_{z}, A \partial_{z}-C \partial_{x}, B \partial_{x}-A \partial_{y}$.

Hence

$$
\partial \cdot \nabla=(P \lambda+Q \mu+R \nu) \cdot\left(\mathfrak{A} \partial_{x}+\mathfrak{W} \partial_{y}+\mathfrak{E} \partial_{z}, \mathfrak{W} \partial_{x}+\mathfrak{B} \partial_{y}+\mathfrak{F} \partial_{z},\left(\mathfrak{)} \partial_{x}+\mathfrak{F} \partial_{y}+\left(\mathfrak{E} \partial_{z} \gamma \lambda, \mu, \nu\right),\right.\right.
$$

viz. coefficient of $\lambda^{2}$

$$
=P \Re \partial_{x}+P \$ \partial_{y}+P\left(j \partial_{z},\right.
$$

and so for the other terms; whence also in $(\partial . \nabla) H$ the coefficients of $\lambda^{2}$, \&c. are

$$
\left(P \mathfrak{A} \partial_{x}+P S_{g} \partial_{y}+P\left(5 \partial_{z}\right) H, \& c .\right.
$$

37. Again, in Jac. $(U, H, \Phi)$, where $\Phi=(\mathfrak{A}, \mathfrak{B}, \mathfrak{E}, \mathfrak{F}, \mathfrak{S} \text {, } \mathfrak{S} \chi \lambda, \mu, \nu)^{2}$, the coefficients of $\lambda^{2}$, \&c. are Jac. $(U, H, \mathfrak{A})$, \&c.; and hence the assumed equation

$$
\text { (ว. } \nabla) H=\text { Jac. }(U, H, \Phi) \text {, }
$$

in regard to the term in $\lambda^{2}$, is

$$
\left(P \mathfrak{A} \partial_{x}+P \mathfrak{S} \partial_{y}+P\left(S \partial_{z}\right) H=J a c .(U, H, \mathfrak{A}),\right.
$$

and we have

$$
\begin{aligned}
& \text { Jac. }(U, H, \mathfrak{l})=\left|\begin{array}{lll}
A, & B, & C \\
\partial_{x} H, & \partial_{y} H, & \partial_{z} H \\
\partial_{x}, & \partial_{y}, & \partial_{z}
\end{array}\right| \text { } \\
& =\left[\partial_{x} H\left(C \partial_{y}-B \partial_{z}\right)+\partial_{y} H\left(A \partial_{z}-C \partial_{x}\right)+\partial_{z} H\left(B \partial_{x}-A \partial_{y}\right)\right] \mathfrak{A}, \\
& =\left(\partial_{x} H \cdot P+\partial_{y} H \cdot Q+\partial_{z} H \cdot R\right) \mathfrak{A} ;
\end{aligned}
$$

so that the equation is

$$
\begin{aligned}
& P \mathfrak{A} \partial_{x} H+P \mathfrak{S} \partial_{y} H+P \mathfrak{S} \partial_{z} H, \\
= & P \mathfrak{A} \partial_{x} H+Q \mathfrak{2} \partial_{y} H+R \mathfrak{A} \partial_{z} H,
\end{aligned}
$$

or, as this may be written,

$$
\begin{gathered}
{\left[\left(B \partial_{z}-C \partial_{y}\right) \mathfrak{S}-\left(\boldsymbol{C} \partial_{x}-A \partial_{z}\right) \mathfrak{A}\right] \partial_{y} H} \\
+\left[\left(B \partial_{z}-C \partial_{y}\right) \mathscr{S}-\left(A \partial_{y}-B \partial_{x}\right) \mathfrak{A}\right] \partial_{z} H=0 .
\end{gathered}
$$


38. The coefficient of $\partial_{y} H$ is

$$
=\quad A \partial_{z} \mathfrak{A}+B \partial_{z} \mathfrak{S}-C\left(\partial_{x} \mathfrak{A}+\partial_{y} \mathfrak{F}\right),
$$

which, in virtue of the identity, post, No. $40, \partial_{x} \mathfrak{A}+\partial_{y} \mathfrak{F}+\partial_{z} \mathscr{S}=0$, is

$$
=\quad A \partial_{z} \mathfrak{A}+\mathfrak{B} \partial_{z} \mathfrak{S}+C \partial_{z} \mathfrak{S} ;
$$

and in like manner the coefficient of $\partial_{z} H$ is

so that the equation is

$$
=-\left(A \partial_{y} \mathfrak{l}+B \partial_{y} \mathfrak{S}+C \partial_{y} \mathscr{S}\right),
$$

$$
\left(A \partial_{z} \mathfrak{A}+B \partial_{z} \mathfrak{S}+C \partial_{z} \mathfrak{S}\right) \partial_{y} H-\left(A \partial_{y} \mathfrak{A}+B \partial_{y} \mathfrak{g}+C \partial_{z}(\mathfrak{S}) \partial_{z} H=0 .\right.
$$

39. But we have

$$
\begin{aligned}
& \mathfrak{A} a+\mathfrak{S} h+\mathfrak{b} g=H, \\
& \mathfrak{A} h+\mathfrak{S} b+\mathfrak{S f} f=0, \\
& \mathfrak{A} g+\mathfrak{H} f+\mathfrak{S} c=0,
\end{aligned}
$$

or multiplying by $x, y, z$ and adding,

whence also

$$
(m-1)(\mathfrak{A} A+\mathfrak{S} B+\mathscr{H} C)=x H ;
$$

that is

$$
(m-1)\left(\mathfrak{A} h+\mathfrak{S} b+\sqrt{5} c+A \partial_{y} \mathfrak{A}+B \partial_{y} \mathfrak{F}+C \partial_{y} \mathbb{S}\right)=x \partial_{y} H,
$$

$$
(m-1)\left(A \partial_{y} \mathfrak{A}+B \partial_{y} \mathfrak{g}+C \partial_{y}(\mathfrak{S})=x \partial_{y} H\right.
$$

and in like manner

$$
(m-1)\left(A \partial_{z} \mathfrak{A}+B \partial_{z} \mathfrak{S}+C \partial_{z} \mathfrak{F}\right)=x \partial_{z} H,
$$

whence the equation in question. The terms in $\lambda^{2}$ are thus shown to be equal, and it might in a similar manner be shown that the terms in $\mu \nu$ are equal; the other terms will then be equal, and we have therefore

$$
\text { (ว. } \nabla) H=\text { Jac. }(U, H, \Phi) \text {. }
$$

40. The identity

$$
\partial_{x} \mathfrak{A}+\partial_{y} \mathfrak{G}+\partial_{z}(\mathfrak{S}=0
$$

assumed in the course of the foregoing proof is easily proved. We have in fact

$$
\begin{gathered}
\partial_{x} \mathfrak{I}+\partial_{y} \mathfrak{F}+\partial_{z} \mathfrak{S}=\partial_{x}\left(b c-f^{2}\right)+\partial_{y}(f g-c h)+\partial_{z}(f h-b g) \\
=b\left(\partial_{x} c-\partial_{x} g\right)+c\left(\partial_{x} b-\partial_{y} h\right)+f\left(-2 \partial_{x} f+\partial_{y} g+\partial_{z} h\right)+g\left(\partial_{y} f-\partial_{z} b\right)+h\left(-\partial_{y} c+\partial_{z} f\right),
\end{gathered}
$$

where the coefficients of $b, c, f, g, h$ separately vanish: we have of course the system

$$
\begin{aligned}
& \partial_{x} \mathfrak{A}+\partial_{y} \mathfrak{F}+\partial_{z} \mathfrak{W}=0, \\
& \partial_{x} \mathfrak{G}+\partial_{y} \mathfrak{B}+\partial_{z} \mathfrak{F}=0, \\
& \partial_{x} \mathfrak{H}+\partial_{y} \mathfrak{F}+\partial_{z} \mathfrak{E}=0 .
\end{aligned}
$$


Article Nos. 41 to 46.-Proof of identities for the fourth transformation.

41. Consider the coefficients $(a, b, c, f, g, h)$ and the inverse set $(\mathfrak{A}, \mathfrak{B}, \mathfrak{C}, \mathfrak{F}, \mathfrak{f}, \mathfrak{S})$, and the coefficients $\left(a^{\prime}, b^{\prime}, c^{\prime}, f^{\prime}, g^{\prime}, h^{\prime}\right)$, and the inverse set $\left(\mathfrak{H}^{\prime}, \mathfrak{B}^{\prime}, \mathfrak{E}^{\prime}, \mathfrak{F}^{\prime}, \mathfrak{E}, \mathfrak{S}^{\prime}\right)$; then we have identically

$$
\begin{aligned}
& (a, \ldots \nmid x, y, z)^{2}\left(\mathfrak{A}^{\prime}, \ldots \nmid(a, \ldots)-\left(\mathfrak{H}^{\prime}, \ldots \chi a x+h y+g z, \ldots\right)^{2}\right. \\
& =\left(a^{\prime}, \ldots \chi x, y, z\right)^{2}\left(\mathfrak{A}, \ldots \chi a^{\prime}, . .\right)-\left(\mathfrak{A}, \ldots \chi a^{\prime} x+h^{\prime} y+g^{\prime} z, . .\right)^{2},
\end{aligned}
$$

where $\left(\mathfrak{I}^{\prime}, \ldots \nmid(a, \ldots)\right.$ and $\left(\mathfrak{A}, \ldots \chi a^{\prime}, \ldots\right)$ stand for

$$
\left(\mathfrak{H}^{\prime}, \mathfrak{B}^{\prime}, \mathfrak{E}^{\prime}, \mathscr{F}^{\prime}, \mathbb{S}^{\prime}, \mathfrak{S}^{\prime} \chi a, b, c, 2 f, 2 g, 2 h\right)
$$

and

$$
\text { ( } \mathfrak{A}, \mathfrak{B}, \mathfrak{C}, \mathfrak{F}, \mathfrak{B}, \mathfrak{S} \gamma\left(a^{\prime}, b^{\prime}, c^{\prime}, 2 f^{\prime}, 2 g^{\prime}, 2 h^{\prime}\right)
$$

respectively.

42. Taking ( $a, b, c, f, g, h$ ), the second differential coefficients of a function $U$ of the order $m$, and in like manner $\left(a^{\prime}, b^{\prime}, c^{\prime}, f^{\prime}, g^{\prime}, h^{\prime}\right)$, the second differential coefficients of a function $U^{\prime}$ of the order $m^{\prime}$, we have

$$
\begin{aligned}
& m(m-1) U \cdot\left(\mathfrak{H}^{\prime}, \ldots \partial_{x}, \partial_{y}, \partial_{z}\right)^{2} U^{\prime}-(m-1)^{2}\left(\mathfrak{H}^{\prime}, \ldots \nmid \partial_{x} U, \partial_{y} U, \partial_{z} U\right)^{2} \\
= & m^{\prime}\left(m^{\prime}-1\right) U^{\prime} \cdot\left(\mathfrak{A}, \ldots \nmid \partial_{x}, \partial_{y}, \partial_{z}\right)^{2} U-\left(m^{\prime}-1\right)^{2}\left(\mathfrak{A}, \ldots \chi \partial_{x} U^{\prime}, \partial_{y} U^{\prime}, \partial_{z} U^{\prime}\right)^{2} ;
\end{aligned}
$$

and in particular if $U^{\prime}$ be the Hessian of $U$, then $m^{\prime}=3 m-6$.

43. Hence writing

$$
\begin{aligned}
& \Omega=\left(\mathfrak{A}, \ldots \nmid \partial_{x}, \partial_{y}, \partial_{z}\right)^{2} H, \quad \Psi=\left(\mathfrak{A}, \ldots \chi \partial_{x} H, \partial_{y} H, \partial_{z} H\right)^{2}, \\
& \Omega_{1}=\left(\mathfrak{H}, \ldots \nmid \partial_{x}, \partial_{y}, \partial_{z}\right)^{2} U, \quad \Psi_{1}=\left(\mathfrak{H}^{\prime}, \ldots \chi \partial_{x} U, \partial_{y} U, \partial_{z} U\right)^{2},
\end{aligned}
$$

we have

$$
m(m-1) U \Omega_{1}-(m-1)^{2} \Psi_{1}=(3 m-6)(3 m-7) H \Omega-(3 m-7)^{2} \Psi ;
$$

or if $U=0$, then

$$
-(m-1)^{2} \Psi_{1}=(3 m-6)(3 m-7) H \Omega-(3 m-7)^{2} \Psi ;
$$

whence also

$$
-(m-1)^{2} \partial \Psi_{1}=(3 m-6)(3 m-7)(H \partial \Omega+\Omega \partial H)-(3 m-7)^{2} \partial \Psi,
$$

which is the formula, ante No. 21.

44. Recurring to the original formula, since this is an actual identity, we may operate on it with the differential symbol $\partial$ on the three assumptions:

1. $(a, b, c, f, g, h),(\mathfrak{H}, \mathfrak{B}, \mathfrak{C}, \mathfrak{F}, \mathfrak{f}, \mathfrak{S})$ are alone variable.

2. $\left(a^{\prime}, b^{\prime}, c^{\prime}, f^{\prime}, g^{\prime}, h^{\prime}\right),\left(\mathfrak{A}^{\prime}, \mathfrak{B}^{\prime}, \mathfrak{b}^{\prime}, \mathfrak{F}^{\prime}, \mathfrak{S}^{\prime}, \mathfrak{S}^{\prime}\right)$ are alone variable.

3. $(x, y, z)$ are alone variable.

c. $\mathbf{v}$. 
We thus obtain

$$
\begin{aligned}
& (\partial a, \ldots \gamma x, y, z)^{2}\left(\mathfrak{A}^{\prime}, \ldots \gamma a, \ldots\right) \\
& =\left(a^{\prime}, \ldots \gamma x, y, z\right)^{2}\left(\partial \mathfrak{A}, \ldots \gamma a^{\prime}, \ldots\right) \\
& -\left(\partial \mathfrak{A}, . . \gamma a^{\prime} x+h^{\prime} y+g^{\prime} z, . .\right)^{2} \\
& +(a, . \gamma \chi x, y, z)^{2}\left(\mathfrak{I}^{\prime}, \ldots \gamma \partial a, \ldots\right) \\
& -2\left(\mathfrak{A}^{\prime}, . . \gamma a x+h y+g z, . . \gamma x \partial a+y \partial b+z \partial c, . .\right) \\
& (a, \ldots \gamma x, y, z)^{2}\left(\partial \mathfrak{A}^{\prime}, \ldots \gamma a, \ldots\right) \\
& =\left(\partial a^{\prime}, \ldots \gamma x, y, z\right)^{2}\left(\mathfrak{A}, \ldots \gamma a^{\prime}, \ldots\right) \\
& +\left(a^{\prime}, \ldots \gamma x, y, z\right)^{2}\left(\mathfrak{A}, \ldots \gamma \partial a^{\prime} . .\right) \\
& -2\left(\mathfrak{A}, . . \gamma a^{\prime} x+h^{\prime} y+g^{\prime} z, . . \chi x \partial a^{\prime}+y \partial h^{\prime}+z \partial g^{\prime}, . .\right) \text {, } \\
& 2(a, . . \chi x, y, z \gamma \partial x, \partial y, \partial z)\left(\mathfrak{H}^{\prime}, . . \chi(a, . .)=2\left(a^{\prime}, . \gamma \chi x, y, z \gamma \partial x, \partial y, \partial z\right)\left(\mathfrak{A}, . . \chi a^{\prime}, . .\right)\right. \\
& -2\left(\mathfrak{I}^{\prime}, . \gamma a x+h y+g z, . \gamma a \partial x+h \partial y+g d z, .\right) \\
& -2\left(\mathfrak{A}, . \gamma a^{\prime} x+h^{\prime} y+g^{\prime} z, . \gamma a^{\prime} \partial x+h^{\prime} \partial y+g^{\prime} \partial z, . .\right) \text {. }
\end{aligned}
$$

45. If in these equations respectively we suppose as before that $(a, b, c, f, g, h)$ are the second differential coefficients of a function $U$ of the order $m$, and $\left(a^{\prime}, b^{\prime}, c^{\prime}, f^{\prime}, g^{\prime}, h^{\prime}\right)$ the second differential coefficients of a function $U^{\prime}$ of the order $m^{\prime}$; and that $(A, B, C)$, $\left(A^{\prime}, B^{\prime}, C^{\prime}\right)$ are the first differential coefficients of these functions respectively, then after some easy reductions we have

$$
\begin{aligned}
& (m-1)(m-2) \partial U\left(\mathfrak{H}^{\prime}, \ldots \gamma a, . .\right) \\
& =m^{\prime}\left(m^{\prime}-1\right) U^{\prime}\left(\partial \mathfrak{A}, . . \gamma a^{\prime}, \ldots\right) \\
& +m(m-1) U\left(\mathfrak{H}^{\prime}, \ldots \gamma \partial a, \ldots\right) \\
& -\left(m^{\prime}-1\right)^{2}\left(\partial \Re, \ldots \gamma A^{\prime}, B^{\prime}, C^{\prime}\right)^{2} \text {, } \\
& -2(m-1)(m-2)\left(\mathfrak{H}^{\prime}, . . \gamma A, B, C \gamma \partial A, \partial B, \partial C\right) \\
& m(m-1) U\left(\partial A^{\prime}, \ldots \gamma a^{\prime}, \ldots\right) \\
& =\left(m^{\prime}-1\right)\left(m^{\prime}-2\right) \partial U^{\prime}\left(\mathfrak{A}, . . \gamma a^{\prime}, . .\right) \\
& +m^{\prime}\left(m^{\prime}-1\right) \bar{U}^{\prime}\left(\mathfrak{A}, \ldots \gamma \partial \alpha^{\prime}, \ldots\right) \\
& -2\left(m^{\prime}-1\right)\left(m^{\prime}-2\right)\left(\mathfrak{A}, . . \chi A^{\prime}, B^{\prime}, C^{\prime} \gamma \partial A^{\prime}, \partial B^{\prime}, \partial C^{\prime}\right) \\
& 2(m-1) \partial U\left(\mathfrak{l}^{\prime}, \ldots \gamma a, \ldots\right) \\
& =2\left(m^{\prime}-1\right) \partial U^{\prime}\left(\mathfrak{A}, \ldots \gamma a^{\prime}, \ldots\right) \\
& -2\left(m^{\prime}-1\right)\left(\mathfrak{A}, . . \gamma A^{\prime}, B^{\prime}, C^{\prime} \chi \partial A^{\prime}, \partial B^{\prime}, \partial \mathbb{E}^{\prime}\right) \text {, }
\end{aligned}
$$

equations which may be verified by remarking that their sum is

$$
\begin{aligned}
& m(m-1)\left\{\partial U\left(\mathfrak{I}^{\prime}, \ldots \gamma a, \ldots\right)+U\left[\left(\mathfrak{H}^{\prime}, \ldots \gamma \partial a, \ldots\right)+\left(\partial \mathfrak{I}^{\prime}, \ldots \gamma a, \ldots\right)\right]\right\} \\
& \left.-(m-1)^{2}\left\{\partial \mathfrak{U}^{\prime}, \ldots \gamma A, B, C\right)^{2}+\left(\mathfrak{A}^{\prime}, \ldots \gamma A, B, C \gamma \partial A, \partial B, \partial C\right)\right\}=m^{\prime}\left(m^{\prime}-1\right) \text { \&c., }
\end{aligned}
$$

viz., this is the derivative with $\partial$ of the equation

$$
m(m-1) U\left(\mathfrak{A}^{\prime}, . \gamma a, \ldots\right)-(m-1)^{2}\left(A^{\prime}, \ldots \gamma A, B, C\right)^{2}=m^{\prime}\left(m^{\prime}-1\right) \& c .
$$

46. Taking now $U^{\prime}=H$, and therefore $m^{\prime}=3 m-6$; putting also $U=0, \partial U=0$, and writing as before

$$
\begin{aligned}
& E \Psi=\left(\partial \mathfrak{A}, \ldots \gamma A^{\prime}, B^{\prime}, C^{\prime}\right)^{2}, \\
& F \Psi=\left(\mathfrak{A}, \ldots \gamma A^{\prime}, B^{\prime}, C^{\prime} \gamma \partial A^{\prime}, \partial B^{\prime}, \partial C^{\prime}\right), \\
& E \Psi_{1}=\left(\partial \mathfrak{I}^{\prime}, \ldots \gamma A, B, \Upsilon^{2},\right. \\
& F^{\prime} \Psi_{1}=(\mathfrak{A}, \ldots \gamma A, B, C \gamma \partial A, \partial B, \partial C), \\
& E \Omega=\left(\partial \mathfrak{A}, . . \gamma a^{\prime}, \ldots\right), \\
& F \Omega=\left(\mathfrak{A}, \ldots \gamma \partial a^{\prime}, . .\right),
\end{aligned}
$$


then the three equations are

$$
\begin{aligned}
-2(m-1)(m-2) F \psi_{1}= & (3 m-6)(3 m-7) H E \Omega-(3 m-7)^{2} E \Psi, \\
-(m-1)^{2} E \Psi & =(3 m-7)(3 m-8) \Omega \partial H \\
& +(3 m-6)(3 m-7) H F \Omega-2(3 m-7)(3 m-8) F^{h} \Psi, \\
-2(m-1) F \Psi_{1} & =2(3 m-7) \Omega \partial H-2(3 m-7) F^{\top} \Psi,
\end{aligned}
$$

whence, adding, we have

$$
\begin{aligned}
-(m-1)^{2}\left(E \Psi_{1}+2 F^{2} \Psi_{1}\right)= & -(3 m-7)^{2}\left(E \Psi+2 F^{\natural} \Psi\right) \\
& +(3 m-6)(3 m-7)\{\Omega \partial H+H(E \Omega+F \Omega)\},
\end{aligned}
$$

(that is

$$
-(m-1)^{2} \partial \Psi_{1} \quad=-(3 m-7)^{2} \partial \Psi+(3 m-6)(3 m-7) \partial . \Omega H,
$$

which is right).

And by linearly combining the three equations, we deduce

$$
\begin{aligned}
& (3 m-6)(3 m-7) H E \Omega=-2(m-1)(m-2) F \Psi_{1} \\
& (3 m-7) \Omega \partial H=-(m-1) \quad F^{\top} \Psi_{1}+(3 m-7) \quad F \Psi, \\
& (3 m-6)(3 m-7) H F \Omega=(m-1)(3 m-8) F^{\top} \Psi_{1}+(3 m-7)(3 m-8) F \Psi-(m-1)^{2} E \Psi_{1},
\end{aligned}
$$

which are the formulæ, ante, No. 24.

Article Nos. 47 to 50.-Proof of an identity used in the fourth transformation, viz.,

$$
\text { Jac. }(U, \bar{\nabla} H, H)=-\frac{m-1}{3 m-7} F^{2} \Psi_{1}
$$

or say

$$
\text { Jac. }(U, H, \bar{\nabla} H)=\frac{m-1}{3 m-7}\left(\mathfrak{H}^{\prime}, . . \chi A, B, C \gamma \partial A, \partial B, \partial C\right) \text {. }
$$

47. We have

$$
\begin{aligned}
& \nabla=\left(\mathfrak{A}, \ldots \gamma \lambda, \mu, \nu \nmid \partial_{x}, \partial_{y}, \partial_{z}\right) \\
& \left.=((\mathfrak{A}, \mathfrak{S}, \mathfrak{B}) \lambda, \mu, \nu),(\mathfrak{S}, \mathfrak{B}, \mathfrak{F} \gamma \lambda, \mu, \nu),(\mathfrak{F}) \mathfrak{F},(\mathfrak{F}) \lambda, \mu, \nu) \gamma \partial_{x}, \partial_{y}, \partial_{z}\right) \text {; }
\end{aligned}
$$

or, attending to the effect of the bar as denoting the exemption of the $(\mathfrak{A}, \ldots)$ from differentiation,

$$
\begin{aligned}
\text { Jac. }(U, H, \bar{\nabla} H)= & (\mathfrak{A}, \mathfrak{F}, \mathfrak{G} \gamma \lambda, \mu, \nu) \text { Jac. }\left(U, H, \partial_{x} H\right) \\
+ & (\mathfrak{S}, \mathfrak{B}, \mathfrak{F} \gamma \lambda, \mu, \nu) \text { Jac. }\left(U, H, \partial_{y} H\right) \\
+ & (\mathfrak{S}, \mathfrak{F}, \mathfrak{\complement} \gamma \lambda, \mu, \nu) \text { Jac. }\left(U, H, \partial_{z} H\right) .
\end{aligned}
$$


48. Now

$$
\text { Jac. }\left(U, H, \partial_{x} H\right)=\frac{1}{3 m-6} \text { Jac. }\left(U, x \partial_{x} H+y \partial_{y} H+z \partial_{z} H, \partial_{x} H\right),
$$

and the last-mentioned Jacobian is

$$
\begin{gathered}
=\partial_{x} H \text { Jac. }\left(U, x, \partial_{x} H\right)+\partial_{y} H \text { Jac. }\left(U, y, \partial_{x} H\right)+\partial_{z} H \text { Jac. }\left(U, z, \partial_{x} H\right) \\
+y \text { Jac. }\left(U, \partial_{y} H, \partial_{x} H\right)+z \text { Jac. }\left(U, \partial_{z} H, \partial_{x} H\right),
\end{gathered}
$$

where the second line is

$$
=-y \text { Jac. }\left(U, \partial_{x} H, \partial_{y} H\right)+z \text { Jac. }\left(U, \partial_{z} H, \partial_{x} H\right),
$$

or writing $\left(A^{\prime}, B^{\prime}, C^{\prime}\right)$ for the first differential coefficients and $\left(a^{\prime}, b^{\prime}, c^{\prime}, f^{\prime}, g^{\prime}, h^{\prime}\right)$ for the second differential coefficients of $H$, this is

$$
\begin{aligned}
& =-y\left|\begin{array}{ccc}
A, & B, & C \\
a^{\prime}, & h^{\prime}, & g^{\prime} \\
h^{\prime}, & b^{\prime}, & f^{\prime}
\end{array}\right|+z\left|\begin{array}{ccc}
A, & B, & C \\
g^{\prime}, & f^{\prime}, & c^{\prime} \\
a^{\prime}, & h^{\prime}, & g^{\prime}
\end{array}\right| \\
& =-y\left(\mathscr{S}^{\prime}, \mathfrak{F}^{\prime}, \mathfrak{S}^{\prime} \gamma A, B, C\right)+z\left(\mathfrak{S}^{\prime}, \mathfrak{B}^{\prime}, \mathfrak{F}^{\prime} \gamma A, B, C\right) \text {. }
\end{aligned}
$$

The first line is

$$
\begin{aligned}
& =\left|\begin{array}{lll}
A, & B, & C \\
A^{\prime}, & B^{\prime}, & C^{\prime} \\
a^{\prime}, & h^{\prime}, & g^{\prime}
\end{array}\right| \\
& =A\left(B^{\prime} g^{\prime}-C^{\prime} i^{\prime}\right)+B\left(C^{\prime} a^{\prime}-A^{\prime} g^{\prime}\right)+C\left(A^{\prime} h^{\prime}-B^{\prime} a^{\prime}\right),
\end{aligned}
$$

or reducing by the formulæ,

this is

$$
(3 m-7)\left(A^{\prime}, B^{\prime}, C^{\prime}\right)=\left(a^{\prime} x+h^{\prime} y+g^{\prime} z, h^{\prime} x+b^{\prime} y+f^{\prime} z, g^{\prime} x+f^{\prime} y+c^{\prime} z\right),
$$

$$
\begin{aligned}
& =\frac{1}{3 m-7}\left\{A\left(-\mathfrak{S}^{\prime} y+\mathfrak{S}^{\prime} z\right)+B\left(-\mathfrak{F}^{\prime} y+\mathfrak{B}^{\prime} z\right)+C\left(-\mathfrak{夭}^{\prime} y+\mathfrak{F}^{\prime} z\right)\right\} \\
& \left.=\frac{1}{3 m-7}\left\{-y\left(\mathscr{H}^{\prime}, \mathfrak{F}^{\prime}, \mathfrak{C}^{\prime}\right\} A, B, C\right)+z\left(\mathfrak{S}^{\prime}, \mathfrak{B}^{\prime}, \mathfrak{F}^{\prime} \mathfrak{X} A, B, C\right)\right\} .
\end{aligned}
$$

Hence we have

Jac. $\left(U, H, \partial_{x} H\right)=\frac{1}{3 m-6}\left(1+\frac{1}{3 m-7}\right)\left\{-y\left(\mathfrak{S}^{\prime}, \mathfrak{F}^{\prime}, \mathfrak{f}^{\prime} \gamma A, B, C\right)+z\left(\mathfrak{S}^{\prime}, \mathfrak{B}^{\prime}, \mathfrak{F}^{\prime} \gamma A, B, C\right)\right\}$

$$
\left.=\frac{1}{3 m-7} \quad\left\{-y\left(\mathscr{H}^{\prime}, \mathfrak{F}^{\prime}, \mathfrak{E}^{\prime}\right\} A, B, C\right)+z\left(\mathfrak{S}^{\prime}, \mathfrak{B}^{\prime}, \mathfrak{F}^{\prime} \gamma A, B, C\right)\right\} ;
$$

and in like manner

Jac. $\left(U, H, \partial_{y} H\right)=\frac{1}{3 m-7}$

$\left.\left\{-z\left(\mathfrak{H}^{\prime}, \mathfrak{S}^{\prime}, \mathfrak{H S}^{\prime}\right\} A, B, C\right)+x\left(\mathfrak{F}^{\prime}, \mathfrak{F}^{\prime},\left(\mathfrak{F}^{\prime}\right\} A, B, C\right)\right\}$,

Jac. $\left(U, H, \partial_{z} H\right)=\frac{1}{3 m-7}$

$\left.\left\{-x\left(\mathfrak{F}^{\prime}, \mathfrak{B}^{\prime}, \mathfrak{F}^{\prime} \gamma A, B, C\right)+y\left(\mathfrak{U}^{\prime}, \mathfrak{S}^{\prime}, \mathscr{J}^{\prime}\right\} A, B, C\right)\right\}$ 
49. We thence have

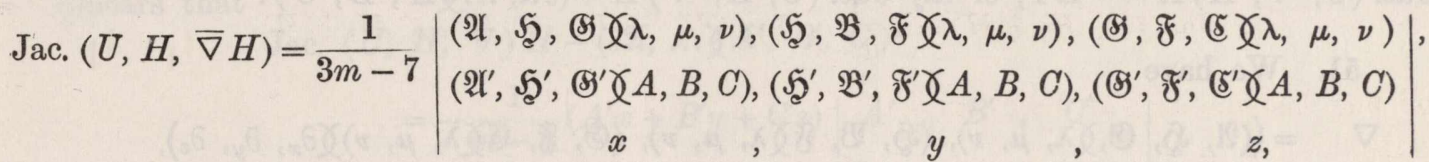

or multiplying the two sides by

$$
H,=\left|\begin{array}{lll}
a, & h, & g \\
h, & b, & f \\
g, & f ; & c
\end{array}\right|
$$

the right-hand side is

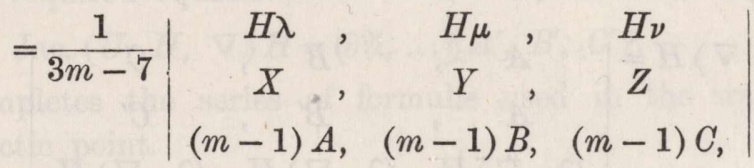

which is

$$
=H \frac{m-1}{3 m-7}\left|\begin{array}{ccc}
\lambda, & \mu, & \nu \\
X, & Y, & Z \\
A, & B, & C,
\end{array}\right|
$$

if for a moment

$$
\begin{aligned}
& X=\left(\mathfrak{A}^{\prime}, . . \gamma A, B, C \gamma a, h, g\right) \text {, } \\
& \boldsymbol{Y}=\left(\mathfrak{A}^{\prime}, . . \gamma A, B, C_{\chi} h, b, f\right) \text {, } \\
& Z=\left(\mathfrak{A}^{\prime}, . . \gamma A, B, c^{\gamma} g, f, c\right) \text {. }
\end{aligned}
$$

50. Hence observing that these equations may be written

$$
\begin{aligned}
& X=\left(\mathfrak{U}^{\prime}, . \gamma A, B, C \gamma \partial_{x} A, \partial_{x} B, \partial_{x} C\right), \\
& Y=\left(\mathfrak{U}^{\prime}, . \gamma A, B, C \gamma \partial_{y} A, \partial_{y} B, \partial_{y} C\right), \\
& Z=\left(\mathfrak{I}^{\prime}, . . \gamma A, B, C \gamma \partial_{z} A, \partial_{z} B, \partial_{z} C\right),
\end{aligned}
$$

and that we have

$$
\partial=\left|\begin{array}{ccc}
\lambda, & \mu, & \nu \\
\partial_{x}, & \partial_{y}, & \partial_{z} \\
A, & B, & C
\end{array}\right|
$$

we obtain for $H$ Jac. $(U, H, \bar{\nabla}, H)$ the value

$$
=H \frac{m-1}{3 m-7}\left(\mathfrak{A}^{\prime}, \ldots \gamma A, B, C_{\chi} \partial A, \partial B, \partial C\right),
$$

or throwing out the factor $H$, we have the required result. 
Article Nos. 51 to 53.-Proof of identity used in the fourth transformation, viz. Jac. $(U, \nabla, H) H=-E \Psi$, or say Jac. $(U, H, \nabla) H=\left(\partial \mathfrak{A}, \ldots \gamma A^{\prime}, B^{\prime}, C^{\prime}\right)^{2}$.

51. We have

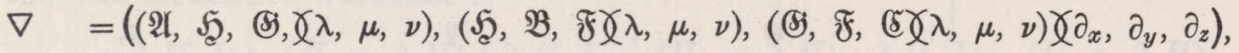
and thence

$\partial_{x} . \nabla=\left(\left(\partial_{x} \mathfrak{H}, \partial_{x} \mathfrak{H}, \partial_{x} \mathfrak{S} \gamma \lambda, \mu, \nu\right),\left(\partial_{x} \mathfrak{H}, \partial_{x} \mathfrak{B}, \partial_{x} \mathfrak{F} \gamma \lambda, \mu, \nu\right),\left(\partial_{x} \mathfrak{S}, \partial_{x} \mathfrak{F}, \partial_{x} \mathfrak{E} \gamma \lambda, \mu, \nu\right) \gamma \partial_{x}, \partial_{y}, \partial_{z}\right)$, and

$\left(\partial_{x} . \nabla\right) H=\left(\left(\partial_{x} \mathfrak{A}, \partial_{x} \mathfrak{S}, \partial_{x}(\mathfrak{S} \gamma \lambda, \mu, \nu),\left(\partial_{x} \mathfrak{F}, \partial_{x} \mathfrak{B}, \partial_{x} \mathfrak{F} \gamma \lambda, \mu, \nu\right),\left(\partial_{x} \mathfrak{S}, \partial_{x} \mathfrak{F}, \partial_{x} \mathfrak{F} \gamma \lambda, \mu, \nu\right) \gamma A^{\prime}, B^{\prime}, C^{\prime}\right)\right.$, with the like values for $\left(\partial_{y} . \nabla\right) H$ and $\left(\partial_{z} . \nabla\right) H$. And then

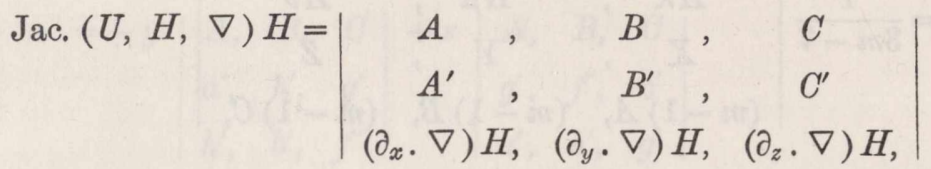

in which the coefficient of $A^{\prime 2}$ is

$$
=\left(C \partial_{y}-B \partial_{z}\right)(\mathfrak{A}, \mathfrak{S}, \text { (S) } \gamma \lambda, \mu, \nu) \text {; }
$$

or putting for shortness

$$
\left(C \partial_{y}-B \partial_{z}, A \partial_{z}-C \partial_{x}, B \partial_{x}-A \partial_{y}\right)=(P, Q, R)
$$

the coefficient is

$$
\text { (Pश, } \mu \mathfrak{S}, P \mathscr{\gamma} \lambda, \mu, \nu) \text {. }
$$

52. We have

$$
\partial=(P \lambda+Q \mu+R \nu)
$$

and thence.

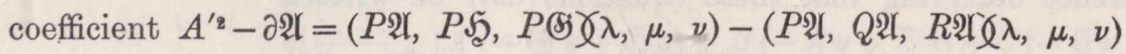

which is

$$
\begin{array}{r}
=\quad \mu\left\{\left(C \partial_{y}-B \partial_{z}\right) \mathfrak{S}-\left(\mathfrak{H} \partial_{z}-\left(\mathfrak{C} \partial_{x}\right) \mathfrak{A}\right\}\right. \\
+\nu\left\{\left(C \partial_{y}-B \partial_{z}\right) \mathfrak{S}-\left(B \partial_{x}-\mathfrak{A} \partial_{y}\right) \mathfrak{R}\right\},
\end{array}
$$

where coefficient of $\mu$ is

$$
\begin{aligned}
& =-A \partial_{z} \mathfrak{H}-B \partial_{z} \mathfrak{H}+C\left(\partial_{x} \mathfrak{H}+\partial_{y} \mathfrak{S}\right) \\
& =-\left(A \partial_{z} \mathfrak{H}+B \partial_{z} \mathfrak{H}+C \partial_{z}(\mathfrak{I )})=-\frac{1}{m-1} x \partial_{z} H\right.
\end{aligned}
$$

and coefficient of $\nu$ is

so that

$$
=+\left(A \partial_{y} \mathfrak{A}+B \partial_{y} \mathfrak{S}+C \partial_{y}(\mathfrak{S})=\frac{1}{m-1} x \partial_{y} H\right.
$$

coefficient $A^{\prime 2}-\partial \mathfrak{U}=-\frac{1}{m-1} x\left(\mu \partial_{z} H-\nu \partial_{y} H\right)$ 
53. By forming in a similar manner the coefficients of the other terms, it appears that

Jac. $(U, H, \nabla) H-\left(\partial \mathfrak{A}, \ldots \gamma A^{\prime}, B^{\prime}, C^{\prime}\right)^{2}$

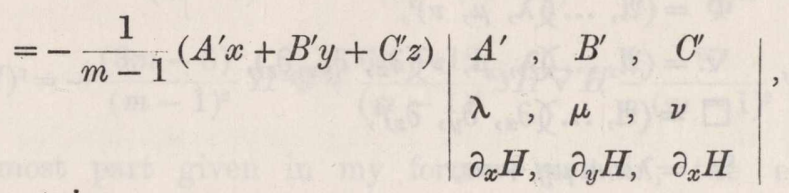

or since the determinant is

$$
\begin{array}{lll}
A^{\prime}, & B^{\prime}, & C^{\prime} \\
\lambda, & \mu, & \nu \\
A^{\prime}, & B^{\prime}, & C^{\prime}
\end{array} \mid,=0,
$$

we have the required equation,

$$
\text { Jac. }(U, H, \nabla) H=\left(\partial \mathfrak{I}, \ldots \gamma A^{\prime}, B^{\prime}, C^{\prime}\right)^{2} \text {. }
$$

This completes the series of formulæ used in the transformations of the condition for the sextactic point.

\section{Appendix, Nos. 54 to 74.}

For the sake of exhibiting in their proper connexion some of the formulæ employed in the foregoing first transformation of the condition for a sextactic point, I have investigated them in the present Appendix, which however is numbered continuously with the memoir.

54. The investigations of my former memoir and the present memoir have reference to the operations

$$
\begin{aligned}
& \partial_{1}=d x \partial_{x}+d y \partial_{y}+d z \partial_{z}, \\
& \partial_{2}=d^{2} x \partial_{x}+d^{2} y \partial_{y}+d^{2} z \partial_{z}, \\
& \partial_{3}=d^{3} x \partial_{x}+d^{3} y \partial_{y}+d^{3} z \partial_{z}, \\
& \& c .,
\end{aligned}
$$

where if $(A, B, C)$ are the first differential coefficients of a function $U=(* \gamma x, y, z)^{m}$, and $\lambda, \mu, \nu$ are arbitrary constants, then we have

so that putting

$$
d x=B \nu-C \mu, \quad d y=C \lambda-A \nu, \quad d z=A \mu-B \lambda
$$

$$
\begin{aligned}
\partial & =(B \nu-C \mu) \partial_{x}+(C \lambda-A \nu) \partial_{y}+(A \mu-B \lambda) \partial_{z} \\
& =\left|\begin{array}{ccc}
A, & B, & C \\
\lambda, & \mu, & \nu \\
\partial_{x}, & \partial_{y}, & \partial_{z},
\end{array}\right|
\end{aligned}
$$

we have $\partial_{1}=\partial$. The foregoing expressions of $(d x, d y, d z)$ determine of course the values of $\left(d^{2} x, d^{2} y, d^{2} z\right),\left(d^{3} x, d^{3} y, d^{3} z\right)$, \&c., and it is throughout assumed that these values are substituted in the symbols $\partial_{2}, \partial_{3}$, \&c., so that $\partial_{1}$, $=\partial$, and $\partial_{2}$, $\partial_{3}$, \&c. denote each of them an operator such as $X \partial_{x}+Y \partial_{y}+Z \partial_{z}$, where $(X, Y, Z)$ are functions of the coordinates; such operator, in so far as it is a function of the coordinates, may therefore be made an operand, and be operated upon by itself or any other like operator. 
55. Taking $(a, b, c, f, g, h)$ for the second differential coefficients of $U,(\mathfrak{H}, \mathfrak{B}, \mathfrak{C}, \mathfrak{F}, \mathbb{E}, \mathfrak{S})$ for the inverse coefficients, and $H$ for the Hessian, I write also

$$
\begin{aligned}
\Phi & =(\mathfrak{A}, \ldots \gamma \lambda, \mu, \nu)^{2}, \\
\nabla & =\left(\mathfrak{A}, \ldots \gamma \lambda, \mu, \nu \gamma\left(\partial_{x}, \partial_{y}, \partial_{z}\right),\right. \\
\square & =\left(\mathfrak{A}, \ldots \gamma\left(\partial_{x}, \partial_{y}, \partial_{z}\right)^{2},\right. \\
\mathfrak{I} & =\lambda x+\mu y+\nu z, \\
\Omega & =\left(\mathfrak{A}, \ldots \gamma \partial_{x}, \partial_{y}, \partial_{z}\right)^{2} H,=\square H, \\
\Psi & =\left(\mathfrak{A}, \ldots \gamma \partial_{x} H, \partial_{y} H, \partial_{z} H\right)^{2}, \\
\Gamma & =\left(a, \ldots \gamma\left(\mu \partial_{z}-\nu \partial_{y}, \nu \partial_{x}-\lambda \partial_{z}, \lambda \partial_{y}-\mu \partial_{x}\right)^{2},\right.
\end{aligned}
$$

and I notice that we have

$$
\begin{array}{ll}
\Gamma U=2 \Phi, & \nabla U=\frac{9}{m-1} H, \quad \square U=3 H, \\
\nabla ৯=\Phi, \quad \nabla^{2} U=H \Phi, \quad \nabla . \partial=0,
\end{array}
$$

the last of which is proved, post No. 65 ; the others are found without any difficulty.

56. I form the Table

$$
\partial_{1} U=0 \text {, }
$$

$\partial_{1}{ }^{2} U=\frac{m U}{m-1} \Phi$

$$
\begin{aligned}
& +\frac{9^{2}}{(m-1)^{2}}(-H), \\
& +\frac{9^{2}}{(m-1)^{2}}(H), \\
& +\frac{9^{2}}{(m-1)^{2}}(-\partial H),
\end{aligned}
$$$$
\partial_{2} U=\frac{m U}{m-1}(-\Phi)
$$

$\partial_{1}{ }^{3} U=\frac{m U}{m-1} \partial \Phi$

$\partial_{1} \partial_{2} U=0$

$$
\begin{aligned}
& \partial_{3} U=\frac{m U}{m-1}(-\partial \Phi) \\
& +\frac{\partial^{2}}{(m-1)^{2}}(\partial H) \\
& \partial_{1}{ }^{4} U=\frac{m U}{m-1}\left(\partial^{2} \Phi-\frac{2 m \Omega}{m-1} \nabla \Phi\right) \\
& +\frac{9^{2}}{(m-1)^{2}}\left(-\partial^{2} H-\frac{3 m-6}{m-1} H \Phi+\frac{29}{m-1} \nabla H\right), \\
& \partial_{1}{ }^{2} \partial_{2} U=\frac{m U}{m-1}\left(\frac{1}{3} \partial_{2} \Phi+\frac{\frac{2}{3} \vartheta}{m-1} \nabla \Phi\right) \\
& +\frac{9^{2}}{(m-1)^{2}}\left(-\frac{1}{3} \partial_{2} H+\frac{m-2}{m-1} H \Phi-\frac{\frac{2}{3} 9}{m-1} \nabla H\right), \\
& \partial_{1} \partial_{3} U=\frac{m U}{m-1}\left(-\frac{1}{3} \partial_{2} \Phi-\Phi_{2}-\frac{\frac{2}{3} \Omega}{m-1} \nabla \Phi\right) \\
& +\frac{9^{2}}{(m-1)^{2}}\left(\frac{1}{3} \partial_{2} H+\frac{1}{m-1} H \Phi+\frac{\frac{2}{3}, 9}{m-1} \nabla H\right) \text {, } \\
& \partial_{2}{ }^{2} U=\frac{m U}{m-1} \\
& +\frac{9^{2}}{(m-1)^{2}}(-H \Phi) \\
& \partial_{4} U=\frac{m U}{m-1}\left(-\frac{2}{3} \partial_{2} \Phi-\partial^{2} \Phi+\Phi^{2}+\frac{\frac{2}{3} \Omega}{m-1} \nabla \Phi\right)+\frac{9^{2}}{(m-1)^{2}}\left(\frac{2}{3} \partial_{2} H+\partial^{2} H-\frac{1}{m-1} H \Phi-\frac{\frac{2}{3} \Omega}{m-1} \nabla H\right) \\
& \partial_{2} H=-\frac{3 m-6}{m-1} H \Phi+\frac{9}{m-1} \nabla H,
\end{aligned}
$$


and assuming $U=0$,

$$
\begin{gathered}
\partial_{1}{ }^{2} H=\partial^{2} H=-\frac{(3 m-6)(3 m-7)}{(m-1)^{2}} H \Phi+\frac{6 m-14}{(m-1)^{2}} \vartheta \nabla H-\frac{9^{2}}{(m-1)^{2}} \Omega, \\
\left(\partial_{1} H\right)^{2}=(\partial H)^{2}=-\frac{(3 m-6)^{2}}{(m-1)^{2}} H^{2} \Phi+\frac{6 m-12}{(m-1)^{2}} 9 H \nabla H-\frac{9^{2}}{(m-1)^{2}} \Psi,
\end{gathered}
$$

which are for the most part given in my former memoir; the expressions for $\partial_{2} U$, $\partial_{3} U$, which are not explicitly given, follow at once from the equations

$$
\left(\partial_{1}{ }^{2}+\partial_{2}\right) U=0, \quad\left(\partial_{1}{ }^{3}+2 \partial_{1} \partial_{2}+\partial_{3}\right) U=0 ;
$$

those for $\partial_{1} \partial_{3} U, \partial_{2}{ }^{2} U$, and $\partial_{4} U$ are new, but when the expressions for $\partial_{1} \partial_{3} U$ and $\partial_{2}{ }^{2} U$ are known, that for $\partial_{4} U$ is at once found from the equation

$$
\left(\partial_{1}{ }^{4}+6 \partial_{1}{ }^{2} \partial_{2}+4 \partial_{1} \partial_{3}+3 \partial_{2}{ }^{2}+\partial_{4}\right) U=0 .
$$

57. Before going further, I remark that we have identically

$$
\begin{aligned}
& (a, \ldots \chi x, y, z)^{2}(a, \ldots \gamma \mu \gamma-\nu \beta, \nu \alpha-\lambda \gamma, \lambda \beta-\mu \alpha)^{2} \\
& -\left|\begin{array}{rrr}
a x+h y+g z, & h x+b y+f z, & g x+f y+c z \\
\lambda & \mu & \nu \\
\alpha \quad, & \beta \quad, & \gamma
\end{array}\right|^{2} \\
& =(\mathfrak{I}, . . \gamma \lambda p-\alpha 9, \mu p-\beta 9, \nu p-\gamma 9)^{2},
\end{aligned}
$$

(if for shortness $p=\alpha x+\beta y+\gamma z, 9=\lambda x+\mu y+\nu z$ )

$$
\begin{aligned}
& =p^{2}(\mathfrak{I}, \ldots \gamma \lambda, \mu, \nu)^{2} \\
& -2 p \text { श (श्l, .. } \gamma \lambda, \mu, \nu \gamma \alpha, \quad \gamma) \\
& +9^{2}\left(\mathfrak{I}, . . \gamma(\alpha, \beta, \gamma)^{2}\right. \text {. }
\end{aligned}
$$

58. If in this equation we take $(a, b, c, f, g, h)$ to be the second differential coefficients of $U$, and write also $(\alpha, \beta, \gamma)=\left(\partial_{x}, \partial_{y}, \partial_{z}\right)$, the equation becomes

$$
\begin{aligned}
m(m-1) U \Gamma-(m-1)^{2} \partial^{2}= & \Phi\left(x \partial_{x}+y \partial_{y}+z \partial_{z}\right)^{2} \\
& -29\left(x \partial_{x}+y \partial_{y}+z \partial_{z}\right) \nabla \\
& +\Phi^{2} \square,
\end{aligned}
$$

which is a general equation for the transformation of $\partial^{2}\left(=\partial_{1}{ }^{2}\right)$.

59. If with the two sides of this equation we operate on $U$, we obtain

$$
\begin{aligned}
m(m-1) U \Gamma U-(m-1)^{2} \partial^{2} U & =m(m-1) \Phi U \\
& -2(m-1) 9 \nabla U \\
& +\quad 92 \square U ;
\end{aligned}
$$

c. V. 
and substituting the values

$$
\Gamma U=2 \Phi, \quad \nabla U=\frac{9}{m-1} H, \quad \square U=3 H,
$$

we find the before-mentioned expression of $\partial_{1}{ }^{2} U$.

60. Operating with the two sides of the same equation on a function $H$ of the order $m^{\prime}$, we find

$$
\begin{aligned}
& m(m-1) U \Gamma H-(m-1)^{2} \partial^{2} H=m^{\prime}\left(m^{\prime}-1\right) \Phi H \\
& -2\left(m^{\prime}-1\right) 9 \nabla H \\
& +\quad 9^{2} \square H \text {; }
\end{aligned}
$$

and in particular if $H$ is the Hessian, then writing $m^{\prime}=3 m-6$, and putting $U=0$, we find the before-mentioned expression for $\partial^{2} H$.

61. But we may also from the general identical equation deduce the expression for $(\partial H)^{2}$. In fact taking $H$ a function of the degree $m^{\prime}$ and writing

$$
(\alpha, \beta, \gamma)=\left(\partial_{x} H, \partial_{y} H, \partial_{z} H\right)
$$

we have

$$
\begin{array}{r}
m(m-1) U\left(a, \ldots \gamma \mu \partial_{z} H-\nu \partial_{y} H, \nu \partial_{x} H-\lambda \partial_{z} H, \lambda \partial_{y} H-\mu \partial_{x} H\right)^{2}-(m-1)^{2}(\partial H)^{2} \\
=m^{\prime 2} \Phi H^{2}-2 m^{\prime} \vartheta H \nabla H+\Upsilon^{2}\left(\mathfrak{R}, \ldots \gamma \partial_{x} H, \partial_{y} H, \partial_{z} H\right)^{2} ;
\end{array}
$$

and if $H$ be the Hessian, then writing $m^{\prime}=3 m-6$ and putting also $U=0$, we find the before-mentioned expression for $(\partial H)^{2}$.

62. Proof of equation

We have

$$
\partial_{2}=-\frac{1}{m-1}\left(x \partial_{x}+y \partial_{y}+z \partial_{z}\right)+\frac{9}{m-1} \nabla
$$

$$
\begin{aligned}
\partial_{2}=\partial . \partial=\left\{(B \nu-C \mu) \partial_{x}+(C \lambda-A \nu) \partial_{y}+(A \mu-B \lambda) \partial_{z}\right\} \\
\left(\lambda\left(C \partial_{y}-B \partial_{z}\right)+\mu\left(A \partial_{z}-C \partial_{x}\right)+\nu\left(B \partial_{x}-A \partial_{y}\right)\right)
\end{aligned}
$$

which is

where

$$
=\lambda\left(C^{\prime} \partial_{y}-B^{\prime} \partial_{z}\right)+\mu\left(A^{\prime} \partial_{z}-C^{\prime} \partial_{x}\right)+\nu\left(B^{\prime} \partial_{x}-A^{\prime} \partial_{y}\right)
$$

$$
\begin{aligned}
A^{\prime}=\partial A & =a(B \nu-C \mu)+h(C \lambda-A \nu)+g(A \mu-B \lambda) \\
& =\lambda(h C-g B)+\mu(g A-a C)+\nu(a B-h A),
\end{aligned}
$$

with the like values for $B^{\prime}$ and $C^{\prime}$. Substituting the values

we have

$$
(m-1)(A, B, C)=(a x+h y+g z, h x+b y+f z, g x+f y+c z),
$$

and similarly

$$
(m-1) A^{\prime}=\lambda(\mathscr{S} y-\mathfrak{S} z)+\mu(\mathfrak{\mho} y-\mathfrak{B} z)+\nu((\mathfrak{夭} y-\mathfrak{F} z)
$$

$$
\begin{aligned}
& (m-1) B^{\prime}=\lambda(\mathfrak{2} z-\mathfrak{S} x)+\mu(\mathfrak{H} z-\mathfrak{F} x)+\nu(\mathfrak{S} z-(\mathfrak{5} x), \\
& (m-1) C^{\prime}=\lambda(\mathfrak{S} x-\mathfrak{2} y)+\mu(\mathfrak{B} x-\mathfrak{S} y)+\nu(\mathfrak{F} x-(\mathfrak{S} y),
\end{aligned}
$$


and then

$$
\begin{aligned}
& (m-1)\left(C^{\prime} \partial_{y}-B^{\prime} \partial_{z}\right)=\lambda\left[\left(\mathfrak{S} x-\mathfrak{2}(y) \partial_{y}-\left(\mathfrak{A} z-(\mathfrak{S} x) \partial_{z}\right]\right.\right. \\
& +\mu\left[(\mathfrak{B} x-\mathfrak{S} y) \partial_{y}-(\mathfrak{S g} z-\mathfrak{F} x) \partial_{z}\right] \\
& +\nu\left[(\sqrt{ } x-\sqrt{ } 5 y) \partial_{y}-\left(\mathfrak{S} z-(\mathfrak{S} x) \partial_{z}\right]\right. \\
& =\lambda\left[x\left(\mathfrak{H}, \mathfrak{S}, \mathfrak{G S} \gamma \partial_{x}, \partial_{y}, \partial_{z}\right)-\mathfrak{A}\left(x \partial_{x}+y \partial_{y}+z \partial_{z}\right)\right] \\
& +\mu\left[x\left(\mathfrak{S}, \mathfrak{B}, \mathfrak{F} \gamma \partial_{x}, \partial_{y}, \partial_{z}\right)-\mathfrak{S}\left(x \partial_{x}+y \partial_{y}+z \partial_{z}\right)\right] \\
& +\nu\left[x\left(\mathfrak{S}, \mathfrak{F},\left(\mathfrak{S} \gamma \partial_{x}, \partial_{y}, \partial_{z}\right)-(\mathfrak{S})\left(x \partial_{x}+y \partial_{y}+z \partial_{z}\right)\right]\right. \\
& =x\left(\mathfrak{R}, \ldots \gamma \lambda, \mu, \nu \gamma \partial_{x}, \partial_{y}, \partial_{z}\right)-(\mathfrak{R}, \mathfrak{S}, \text { (5) } \chi \lambda, \mu, \nu)\left(x \partial_{x}+y \partial_{y}+z \partial_{z}\right) \text {; }
\end{aligned}
$$

that is

$$
(m-1)\left(C^{\prime} \partial_{y}-B^{\prime} \partial_{z}\right)=x \nabla-(\mathfrak{A}, \mathfrak{S}, \text { (j) } \gamma \lambda, \mu, \nu)\left(x \partial_{x}+y \partial_{y}+z \partial_{z}\right),
$$

and so

$$
\begin{aligned}
& (m-1)\left(A^{\prime} \partial_{z}-C^{\prime} \partial_{x}\right)=y \nabla-(\mathfrak{H}, \mathfrak{B}, \mathfrak{F} \gamma \lambda, \mu, \nu)\left(x \partial_{x}+y \partial_{y}+z \partial_{z}\right), \\
& (m-1)\left(B^{\prime} \partial_{x}-A^{\prime} \partial_{y}\right)=z \nabla-(\mathfrak{S}, \mathfrak{F}, \mathfrak{\complement} \gamma \lambda, \mu, \nu)\left(x \partial_{x}+y \partial_{y}+z \partial_{z}\right) ;
\end{aligned}
$$

whence

or finally

$$
\begin{aligned}
(m-1) \partial_{2} & =(\lambda x+\mu y+\nu z) \nabla-(\mathfrak{H}, \ldots \gamma \lambda \lambda, \mu, \nu)^{2} \quad\left(x \partial_{x}+y \partial_{y}+z \partial_{z}\right), \\
& =9 \nabla-\Phi\left(x \partial_{x}+y \partial_{y}+z \partial_{z}\right)
\end{aligned}
$$

$$
\partial_{2}=-\frac{1}{m-1} \Phi\left(x \partial_{x}+y \partial_{y}+z \partial_{z}\right)+\frac{9}{m-1} \nabla .
$$

63. This leads to the expression for $\partial_{2}{ }^{2} U$; we have

$$
\begin{aligned}
\partial_{2}{ }^{2}= & \frac{1}{(m-1)^{2}} \Phi^{2}\left(x \partial_{x}+y \partial_{y}+z \partial_{z}\right)^{2} \\
& -\frac{29}{(m-1)^{2}} \Phi \nabla\left(x \partial_{x}+y \partial_{y}+z \partial_{z}\right) \\
& +\frac{9^{2}}{(m-1)^{2}} \nabla^{2}
\end{aligned}
$$

and operating herewith on $U$, we find

$$
\begin{aligned}
\partial_{2}^{2} U= & \frac{m(m-1)}{(m-1)^{2}} \Phi^{2} U \\
& -\frac{2(m-1) 9}{(m-1)^{2}} \Phi \nabla U \\
& +\frac{9^{2}}{(m-1)^{2}} \nabla^{2} U
\end{aligned}
$$


or since

$$
\nabla U=\frac{\vartheta}{m-1} H, \quad \nabla^{2} U=H \Phi,
$$

this is

$$
\partial_{2}{ }^{2} U=\frac{m U}{(m-1)^{2}} \Phi^{2}+\frac{9^{2}}{(m-1)^{2}} H \Phi .
$$

64. We have $\partial_{1} \partial_{2} U=0$, and thence

that is

$$
\left(\partial_{1}^{2} \partial_{2}+\partial_{1} \partial_{3}+\partial_{2}^{2}\right) U=0
$$

$$
\partial_{1} \partial_{3} U=-\partial_{2}^{2} \partial_{2} U-\partial_{2}{ }^{2} U \text {; }
$$

or substituting the values of $\partial_{1}{ }^{2} \partial_{2} U$ and $\partial_{2}{ }^{2} U$, we find the value of $\partial_{1} \partial_{3} U$ as given in the Table. And then from the equation

$$
\left(\partial_{1}^{4}+6 \partial_{1}{ }^{2} \partial_{2}+4 \partial_{1} \partial_{3}+3 \partial_{2}{ }^{2}+\partial_{4}\right) U=0
$$

or

$$
\partial_{4} U=-\left(\partial_{1}{ }^{4}+6 \partial_{1}{ }^{2} \partial_{2}+4 \partial_{1} \partial_{3}+3 \partial_{2}{ }^{2}\right) U
$$

we find the value of $\partial_{4} U$, and the proof of the expressions in the Table is thus completed.

65. Proof of equation $\nabla . \partial=0$.

We have

and then

$$
\begin{aligned}
\nabla . \partial & =\nabla \cdot\left((B \nu-C \mu) \partial_{x}+(C \lambda-A \nu) \partial_{y}+(A \mu-B \lambda) \partial_{z}\right) \\
& =\nabla \cdot\left(A\left(\mu \partial_{z}-\nu \partial_{y}\right)+B\left(\nu \partial_{x}-\lambda \partial_{z}\right)+C\left(\lambda \partial_{y}-\mu \partial_{x}\right)\right) \\
& =\nabla A\left(\mu \partial_{z}-\nu \partial_{y}\right)+\nabla B\left(\nu \partial_{x}-\lambda \partial_{z}\right)+\nabla C\left(\lambda \delta_{y}-\mu \partial_{x}\right)
\end{aligned}
$$

$$
\begin{aligned}
& \nabla A=(\mathfrak{A}, \ldots \gamma \lambda, \mu, \nu \gamma a, h, g)=H \lambda, \\
& \nabla B=(\mathfrak{A}, \ldots \gamma \lambda, \mu, \nu \gamma h, b, f)=H \mu, \\
& \nabla C=(\mathfrak{A}, \ldots \gamma \lambda, \mu, \nu \gamma g, f, c)=H \nu ;
\end{aligned}
$$

or substituting these values, we have the equation in question.

66. Proof of the expression for $\partial_{3}$.

We have

$$
\partial_{2}=-\frac{1}{m-1} \Phi\left(x \partial_{x}+y \partial_{y}+z \partial_{z}\right)+\frac{9}{m-1} \nabla
$$

and thence operating on the two sides respectively with $\partial_{1},=\partial$, we have

$$
\begin{aligned}
\partial_{3}= & -\frac{1}{m-1}\left\{\partial \Phi\left(x \partial_{x}+y \partial_{y}+z \partial_{z}\right)+\Phi \partial \cdot\left(x \partial_{x}+y \partial_{y}+z \partial_{z}\right)\right\} \\
& +\frac{1}{m-1}\{\partial \Im \nabla+9 \partial \cdot \nabla\} ;
\end{aligned}
$$


or since

this is

$$
\partial .\left(x \partial_{x}+y \partial_{y}+z \partial_{z}\right)=\partial, \partial 9=0,
$$

$$
\partial_{3}=-\frac{1}{m-1} \partial \Phi\left(x \partial_{x}+y \partial_{y}+z \partial_{z}\right)-\frac{1}{m-1} \Phi \partial+\frac{9}{m-1} \partial . \nabla .
$$

67. Proof of expression for $\partial_{3} H$.

Operating with $\partial_{3}$ upon $H$, we have at once

$$
\partial_{3} H=-\frac{3 m-6}{m-1} H \partial \Phi-\frac{1}{m-1} \Phi \partial H+\frac{9}{m-1}(\partial . \nabla) H .
$$

The remainder of the present Appendix is preliminary, or relating to the investigation of the expressions for $\partial_{1} \partial_{2}{ }^{2} U$ and $\partial_{1}{ }^{2} \partial_{3} U$, used ante, No. 31 .

68. Proof of equation $\nabla^{2} \partial U \doteq \Phi \partial H-H \partial \Phi$.

We have identically

$$
\begin{gathered}
(\mathfrak{R}, \ldots \gamma \lambda, \mu, \nu)^{2}\left(\mathfrak{R}, \ldots \backslash \partial_{x}, \partial_{y}, \partial_{z}\right)^{2}-\left[\left(\mathfrak{R}, \ldots \gamma \lambda, \mu, \nu \gamma \partial_{x}, \partial_{y}, \partial_{z}\right)\right]^{2} \\
=(a b c-\& c .)\left(a, \ldots \chi \nu \partial_{y}-\mu \partial_{z}, \lambda \partial_{z}-\nu \partial_{x}, \mu \partial_{x}-\lambda \partial_{y}\right)^{2} ; \\
\Phi \square-\nabla^{2}=H \Gamma ;
\end{gathered}
$$

that is

and then multiplying by $\partial$, and with the result operating on $U$, we find

$$
\Phi \square \partial U-\nabla^{2} \partial U=H \Gamma \partial U .
$$

Now

and thence

$$
\begin{aligned}
\square U & =\left(\mathfrak{I}, \ldots \chi \partial_{x}, \partial_{y}, \partial_{z}\right)^{2} U \\
& =(\mathfrak{I}, \ldots \nmid(a, b, c, 2 f, 2 g, 2 h) ;
\end{aligned}
$$

and observing that

$$
\square \partial U=(\mathfrak{R}, \ldots \chi \gamma \partial a, \partial b, \partial c, 2 \partial f, 2 \partial g, 2 \partial h) ;
$$

and thence that

$$
H=\left|\begin{array}{lll}
a, & h, & g \\
h, & b, & f \\
g, & f, & c
\end{array}\right|,
$$

$$
\begin{aligned}
& \partial H=\left|\begin{array}{rrr}
\partial a, & \partial h, & \partial g \\
h, & b, & f \\
g, & f, & c
\end{array}\right|+\left|\begin{array}{rrr}
a, & h, & g \\
\partial h, & \partial b, & \partial f \\
g, & f, & c
\end{array}\right|+\left|\begin{array}{rrr}
a, & h, & g \\
h, & b, & f \\
\partial g, & \partial f, & \partial c
\end{array}\right|, \\
& =(\mathfrak{A}, \mathfrak{F}, \mathfrak{F} \gamma \partial a, \partial h, \partial g)+(\mathfrak{L}, \mathfrak{B}, \mathfrak{F} \zeta \partial h, \partial b, \partial f)+(\mathfrak{F}, \mathfrak{F},(\mathfrak{b}) \partial g, \partial f, \partial c), \\
& =(\mathfrak{I}, \ldots \nmid \partial a, \partial b, \partial c, 2 \partial f, 2 \partial g, 2 \partial h,
\end{aligned}
$$

we see that

$$
\square \partial U=\partial H \text {. }
$$


Moreover

and thence

$$
\begin{aligned}
\Gamma U= & \left(a, \ldots \gamma \nu \partial_{y}-\mu \partial_{z}, \ldots\right)^{2} U \\
= & a\left(b \nu^{2}+c \mu^{2}-2 f \mu \nu\right) \\
& +b\left(c \lambda^{2}+a \nu^{2}-2 g \nu \lambda\right) \\
& +c\left(a \mu^{2}+b \lambda^{2}-2 h \lambda \mu\right) \\
& +2 f\left(-f \lambda^{2}+g \lambda \mu+h \lambda \nu-a \mu \nu\right) \\
& +2 g\left(f \lambda \mu-g \mu^{2}+h \mu \nu-b \nu \lambda\right) \\
& +2 h\left(f \nu \lambda+g \nu \mu-h \nu^{2}-c \lambda \mu\right)
\end{aligned}
$$

that is

$$
\begin{aligned}
\mathrm{I} \partial U= & \left(a, \ldots \gamma \nu \partial_{y}-\mu \partial_{z}, \ldots\right)^{2} \partial U, \\
= & a\left(\nu^{2} \partial b+\mu^{2} \partial c-2 \mu \nu \partial f\right) \\
& +\& c . \\
= & \lambda^{2}(b \partial c+c \partial b-2 f \partial f) \\
& +\& c . \\
= & (\partial \mathfrak{R}, \partial \mathfrak{B}, \partial \mathfrak{c}, \partial \mathfrak{F}, \partial \mathfrak{s}, \partial \mathfrak{g} \gamma \lambda, \mu, \nu)^{2},
\end{aligned}
$$

Hence the equation

$$
\Gamma \partial U=\partial \Phi .
$$

becomes

\section{$\Phi \square \partial U-\nabla^{2} \partial U=H \Gamma \partial U$}

$$
\Phi \partial H-\nabla^{2} \partial U=H \partial \Phi,
$$

$$
\nabla^{2} \partial U=\text { Фว }-H \partial \Phi .
$$

69. Proof of equation $\partial_{1} \partial_{2}{ }^{2} U=\frac{\lambda^{2}}{(m-1)^{2}}(\Phi \partial H-H \partial \Phi)$.

We have

$$
\begin{aligned}
\partial_{2}{ }^{2}= & \frac{1}{(m-1)^{2}} \Phi^{2}\left(x \partial_{x}+y \partial_{y}+z \partial_{z}\right)^{2} \\
& -\frac{22}{(m-1)^{2}} \Phi\left(x \partial_{x}+y \partial_{y}+z \partial_{z}\right) \nabla \\
& +\frac{\lambda^{2}}{(m-1)^{2}} \nabla^{2}
\end{aligned}
$$

and thence multiplying by $\partial_{1},=\partial$, and with the result operating upon $U$, we find

$$
\partial_{1} \partial_{2}^{2} U=\frac{(m-1)(m-2)}{(m-1)^{2}} \Phi^{2} \partial U-\frac{2(m-2)}{(m-1)^{2}} 2 \Phi \partial \nabla U+\frac{\vartheta^{2}}{(m-1)^{2}} \partial \nabla^{2} U .
$$

But $\partial U=0$, and thence also $\nabla(\partial U)=0$, that is $(\nabla . \partial) U+\nabla \partial U=0$; moreover $\nabla . \partial=0$, and therefore $(\nabla . \partial) U=0$, whence also $\nabla \partial U=0$. Therefore

$$
\partial_{1} \partial_{2}{ }^{2} U=\frac{و^{2}}{(m-1)^{2}} \partial \nabla^{2} U
$$

or substituting for $\partial \nabla^{2} U$ its value $=\Phi \partial H-H \partial \Phi$, we have the required expression for $\partial_{1} \partial_{2}{ }^{2} U$. 
70. Proof of equation

We have

$$
\partial_{1}^{2} \partial_{3} U=\frac{\partial^{2}}{(m-1)^{3}}((3 m-6) H \partial \Phi+(-m+3) \Phi \partial H)+\frac{y^{3}}{(m-1)^{3}}\{-(\partial . \nabla) H\} .
$$

$$
\partial_{3}=-\frac{1}{m-1} \partial \Phi\left(x \partial_{x}+y \partial_{y}+z \partial_{z}\right)-\frac{1}{m-1} \Phi \partial+\frac{2}{m-1} \partial . \nabla
$$

and thence multiplying by $\partial_{1}{ }^{2}=\partial^{2}$, and operating on $U$,

$$
\partial_{1}^{2} \partial_{3} U=-\frac{m-2}{m-1} \partial \Phi \partial^{2} U-\frac{1}{m-1} \Phi \partial^{3} U+\frac{9}{m-1}(\partial . \nabla) \partial^{2} U .
$$

To reduce $(\partial . \nabla) \partial^{2} U$, we have

$$
\begin{aligned}
\partial\left(\nabla \partial^{2} U\right) & =\nabla \partial^{3} U+\left(\partial . \nabla \partial^{2}\right) U, \\
& =\nabla \partial^{3} U+\left[(\partial . \nabla) \partial^{2}+\nabla\left(\partial . \partial^{2}\right)\right] U, \\
& =\nabla \partial^{3} U+(\partial . \nabla) \partial^{2} U+2 \nabla \partial \partial_{2} U,
\end{aligned}
$$

and since

$$
\partial_{2}=-\frac{1}{m-1} \Phi\left(x \partial_{x}+y \partial_{y}+z \partial_{z}\right)+\frac{9}{m-1} \nabla
$$

multiplying by $\nabla \partial$, and with the result operating on $U$, we obtain

$$
\nabla \partial \partial_{2} U=-\frac{m-2}{m-1} \Phi \nabla \partial U+\frac{2}{m-1} \nabla^{2} \partial U
$$

or since $\nabla \partial U=0$, this is

$$
\nabla \partial \partial_{2} U=\frac{\mathcal{Q}}{m-1} \nabla^{2} \partial U
$$

Hence

$$
\partial\left(\nabla \partial^{2} U\right)=\nabla \partial^{3} U+(\partial . \nabla) \partial^{2} U+\frac{2 \lambda}{m-1} \nabla^{2} \partial U
$$

that is

$$
(\partial . \nabla) \partial^{2} U=\partial\left(\nabla \partial^{2} U\right)-\nabla \partial^{3} U-\frac{2 A}{m-1} \nabla^{2} \partial U
$$

Substituting this value of $(\partial . \nabla) \partial^{2} U$, we find

$$
\begin{aligned}
\partial_{1}^{2} \partial_{3} U= & -\frac{m-2}{m-1} \partial \Phi \partial^{2} U-\frac{1}{m-1} \Phi \partial^{3} U \\
& +\frac{\lambda}{m-1}\left(\partial\left(\nabla \partial^{2} U\right)-\nabla \partial^{3} U\right) \\
& +\frac{\vartheta^{2}}{(m-1)^{2}}\left(-2 \nabla^{2} \partial U\right)
\end{aligned}
$$

the three lines whereof are to be separately further reduced. 
71. For the first line we have

and hence

$$
\partial^{2} U=-\frac{\vartheta^{2}}{(m-1)^{2}} H, \quad \partial^{3} U=-\frac{\mathscr{Q}^{2}}{(m-1)^{2}} \partial H,
$$

$$
\text { first line of } \partial_{1}^{2} \partial_{3} U=\frac{\grave{2}^{2}}{(m-1)^{3}}((m-2) H \partial \Phi+\Phi \partial H) \text {. }
$$

72. For the second line, we have

$$
\begin{aligned}
\nabla\left(\partial^{2} U\right) & =\nabla \partial^{2} U+2(\nabla . \partial) \partial U \\
& =\nabla \partial^{2} U, \text { since } \nabla . \partial=0, \text { and therefore }(\nabla . \partial) \partial U=0 ;
\end{aligned}
$$

that is

$$
\begin{aligned}
\nabla \partial^{2} U=\nabla\left(\partial^{2} U\right) & =\nabla\left(\frac{m U}{m-1} \Phi-\frac{9^{2}}{(m-1)^{2}} H\right) \\
& =\frac{m}{m-1}(U \nabla \Phi+\Phi \nabla U)-\frac{1}{(m-1)^{2}}\left(\Upsilon^{2} \nabla H+2 ৯ H \nabla \uparrow\right)
\end{aligned}
$$

or writing

$$
U=0, \quad \nabla U=\frac{\vartheta}{m-1} H, \quad \nabla \partial=\Phi
$$

this is

whence also

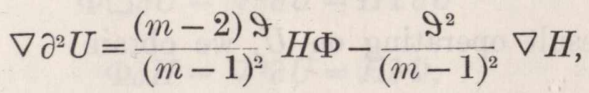

Similarly

$$
\partial\left(\nabla \partial^{2} U\right)=\frac{(m-2) \Phi}{(m-1)^{2}}(H \partial \Phi+\Phi \partial H)-\frac{\AA^{2}}{(m-1)^{2}} \partial(\nabla H) .
$$

$$
\begin{aligned}
\nabla \partial^{3} U & =\nabla\left(\partial^{3} U\right) \\
& =\nabla\left(\frac{m U}{m-1} \partial \Phi-\frac{9^{2}}{(m-1)^{2}} \partial H\right) \\
& =\frac{m}{m-1}(\nabla U \partial \Phi+U \nabla(\partial \Phi))-\frac{1}{(m-1)^{2}}\left(9^{2} \nabla(\partial H)+2 ৯ \nabla \gg \partial H\right)
\end{aligned}
$$

or putting

$$
U=0, \quad \nabla U=\frac{9}{m-1} H, \quad \nabla \vartheta=\Phi,
$$

and observing also that $\nabla(\partial H),=\nabla \partial H+(\nabla . \partial) H$ is equal to $\nabla \partial H$, that is to $\partial \nabla H$, we obtain

$$
\nabla \partial^{3} U=\frac{9}{(m-1)^{2}}(m H \partial \Phi-2 \Phi \partial H)-\frac{9^{2}}{(m-1)^{2}} \partial \nabla H
$$

and then from the above value of $\partial\left(\nabla \partial^{2} U\right)$, we find

$$
\partial\left(\nabla \partial^{2} U\right)-\nabla \partial^{3} U=\frac{9}{(m-1)^{2}}(-2 H \partial \Phi+m \Phi \partial H)+\frac{\mathfrak{I}^{2}}{(m-1)^{2}}(-\partial(\nabla H)+\partial \nabla H)
$$


or observing that the term multiplied by $\frac{9^{2}}{(m-1)^{2}}$ is $=-(\partial . \nabla) H$, we find second line of $\left.\partial_{1}^{2} \partial^{3} U=\frac{9^{2}}{(m-1)^{3}}(-2 H \partial \Phi+m \Phi \partial H)+\frac{\partial^{3}}{(m-1)^{3}}(-\partial . \nabla) H\right)$.

73. For the third line, substituting for $\nabla^{2} \partial U$ its value $=\Phi \partial H-H \partial \Phi$, we have third line of $\partial_{1}{ }^{2} \partial_{3} U=-\frac{29^{2}}{(m-1)^{2}}(\Phi \partial H-H \partial \Phi)$.

74. Hence, uniting the three lines, we have

$$
\begin{aligned}
\partial_{1}^{2} \partial_{3} U= & \frac{9^{2}}{(m-1)^{3}}((m-2) H \partial \Phi+\quad \Phi \partial H) \\
& +\frac{9^{2}}{(m-1)^{3}}(\quad-2 H \partial \Phi+\quad m \Phi \partial H)+\frac{9^{3}}{(m-1)^{3}}(-(\partial . \nabla) H) \\
& +\frac{9^{2}}{(m-1)^{3}}((2 m-2) H \partial \Phi+(-2 m+2) \Phi \partial H),
\end{aligned}
$$

and, reducing, we have the above-mentioned value of $\partial_{1}{ }^{2} \partial_{3} U$.

c. $v$. 